\title{
Misconceptions about colour categories
}

\section{Abstract}

The origin of colour categories and their relationship to colour perception have been the prime example for testing the influence of language on perception and thought (Sapir-Whorf hypothesis) and more generally for investigating the biological, ecological and cultural determination of human cognition (nature-nurture debate). These themes are central to a broad range of disciplines, including vision research, neuroscience, cognitive psychology, developmental science, cultural anthropology, linguistics, computer science, and philosophy. Unfortunately, though, it has been tacitly taken for granted that the conceptual assumptions and methodological practices from the dawn of empirical research on colour categorisation are the gold standard for current colour category research. Here, we show that these assumptions and practices are obsolete and flawed and have led to four fundamental and widespread misconceptions about colour categorisation: 1.) that colour perception is inherently categorical; 2.) that English Basic Colour Terms correspond to universal categories that are the end point of a fixed evolutionary sequence; 3.) that the prototypes of English Basic Colour Terms are perceptually salient and qualify as focal colours; and 4.) that colour category research essentially revolves around the universalism-realism debate. State-of-theart research on colour categorisation provides new, more sophisticated approaches and allows for rectifying those four statements. At the same time, some of the questions underlying those statements are not convincingly answered yet and constitute major challenges to future research. The critical considerations on colour categorisation may be transferred to research on other kinds of perceptual categorisation to inspire new, more general research questions.

Keywords: Categorical perception, colour vision, colour categories, NatureNurture, Sapir-Whorf-Hypothesis.

\section{Introduction}

Where do perceptual categories come from, and how do they influence the way we perceive the world? Colour categories have been considered to be a prime example for investigating this fundamental question because there is an obvious gap between colour perception and colour categorisation (R. W. Brown \& Lenneberg, 1954). On the one hand, we perceive a multitude of about 2 million different colours (Linhares, Pinto, \& Nascimento, 2008; but the precise numbers is not known, see e.g. Masaoka, Berns, Fairchild, \& Moghareh, 2013). These perceivable colours vary continuously in their hue, saturation, and lightness (cf. Figure 1.a; for details see e.g. Fairchild, 2013; Hunt \& Pointer, 2011). On the other hand, we do not make metric evaluations of hue, saturation and brightness when we communicate about colours. Instead, we name colours through colour terms that refer to more or less discrete colour categories (cf. Figure 1.b). These 


\section{Misconceptions about colour categories}

colour categories are the ensembles of different colour shades that are grouped under a colour term. For example, the colour term "brown" describes a whole range of (brownish) colour shades.

The explanatory gap between colour perception and colour categories provides a particular opportunity to investigate the general link between language and perception. For this reason, colour categories have been at the core of research on linguistic relativity (Berlin \& Kay, 1969; R. W. Brown \& Lenneberg, 1954; Gumperz \& Levinson, 1991; Lenneberg, 1953; Lucy, 1997a). According to this idea, language determines which examples are grouped in a category, and the resulting categories influence the way we perceive and think. This idea is at the core of the Sapir-Whorf hypothesis (Wolff \& Holmes, 2011), which is named after two linguists who originally proposed and elaborated these ideas (Sapir, 1912; Whorf, 1956).

The question of whether language influences perception brought colour categories also into the spotlight of the debate about the cognitive penetrability of perception (Collins \& Olson, 2014; Firestone \& Scholl, 2016). Cognition consists of nonperceptual aspects of mental activities, such as language, memory, and learning. Cognitive penetration means that cognition shapes (or "penetrates") perception (Pylyshyn, 1999). More generally, the Sapir-Whorf Hypothesis as well as questions about cognitive penetrability is strongly related to the Nature-Nurture debate. This debate is about the question of whether human perception is shaped by biologically innate determinants (Nature), or by constraints in the culture and environment of human observers (Nurture).

Colour categories have a dual character that comes with a two-fold challenge to category research. On the one hand, colour categories may be understood as perceptual categories because they categorise a perceptual attribute, namely colour. The term perceptual attribute refers to properties that are the result of perception. As a consequence, the study of colour categories requires understanding colour perception.

It is important to note that the properties of colour perception do not simply reflect a physical property, such as the wavelengths of light. This is illustrated by three of the most fundamental properties of human colour vision: trichromacy, colour opponency and colour constancy (for review see Gegenfurtner \& Kiper, 2003; 


\section{Misconceptions about colour categories}

Witzel \& Gegenfurtner, in press). Trichromacy refers to the fact that human colour vision is three-dimensional (e.g. Brainard, 2015; Gegenfurtner \& Kiper, 2003; Kelber \& Osorio, 2010). Colour opponency implies that there are antagonistic perceptual mechanisms, resulting in opponent colours (e.g. red and green) that mutually exclude and cancel each other (e.g. Gegenfurtner \& Kiper, 2003; Shevell \& Martin, 2017; Wuerger \& Xiao, 2014). Colour constancy describes the ability to identify the colour of a surface across different illuminations despite the fact that the wavelength composition of the light that reaches the eye (the proximal stimulus) strongly changes across illuminations (Foster, 2011; Witzel \& Gegenfurtner, in press). Trichromacy, opponency, and colour constancy are not physical properties of the light that reaches the eye, but rather a result of perceptual processing.

The idea that colour is a perceptual rather than a physical attribute might seem surprising because we experience colour as a property of objects in our environment. However, this may be explained by distal attribution. Distal attribution describes the idea that proximal patterns of stimulation are attributed to an exterior and distinct object (Auvray, Hanneton, Lenay, \& O'Regan, 2005; Hartcher-O'Brien \& Auvray, 2014). In the case of colour perception, distal attribution means that the stimulation in the retina is subjectively experienced as a property of object surfaces and materials. So, colour categories are perceptual categories in the sense that they categorise a perceptual attribute.

On the other hand, colour categories may also be understood as linguistic categories because they correspond to the colour terms used in colour naming. In particular, colour naming involves a linguistic reference (Witzel, 2012). Consider Figure 1.c: a colour term (e.g. "blue") refers to a particular colour (e.g. a shade of blue) through a category (blue). In this triangle of reference, the colour term works as a signifier (linguistic sign), and the particular colour shade is the referent (or object or significate). The concept of a colour category that relates the colour term to the colour constitutes the concept or signified and is the meaning of the colour term (de Saussure, 1972; Eco, 1988; Ogden \& Richards, 1923). Hence, understanding the origin of colour categories links a fundamental perceptual attribute (colour) to language (colour naming). Establishing this link constitutes a 


\section{Misconceptions about colour categories}

second challenge because it requires understanding the cultural, historical and environmental constraints that shape colour naming.

Consequently, the origin of colour categorisation has been investigated by a broad range of disciplines including vision research, cognitive psychology, neuroscience, comparative biology, developmental science, cultural anthropology, linguistics, computer science, philosophy, and history (example references for each of these disciplines may be found below). However, the communication across disciplines and research traditions lags behind the most recent developments within each discipline. Ideas from the early phases of colour category research may still be found in reviews on the topic despite the availability of new findings that challenge those ideas and make them obsolete. Due to their persistence in the scientific literature these ideas prevail in the dissemination across disciplines and to the non-scientific public, leading to misconceptions about colour categorisation (specific examples will be given below). Four major ideas have led to such misconceptions in various sources of literature: categorical perception, Basic Colour Terms, focal colours, and the universalism-relativism debate. The four main sections below will update and critically analyse the state-of-the art on these concepts. The outlook in the last section briefly discusses how the questions and challenges of colour category research may inform and inspire research on other perceptual categories.
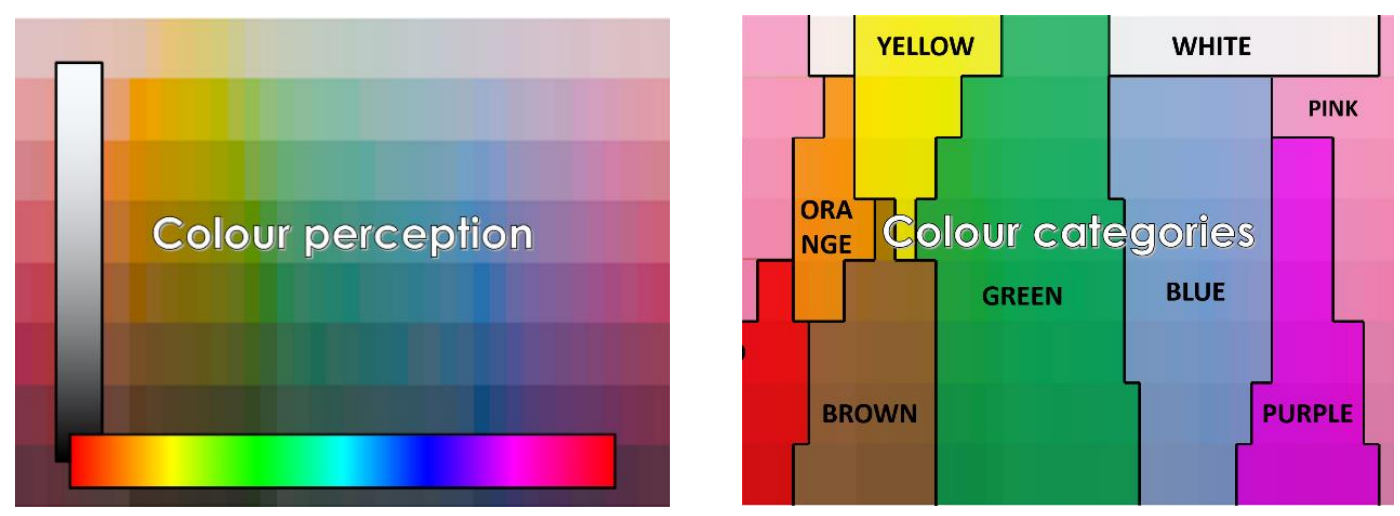


\section{Misconceptions about colour categories}

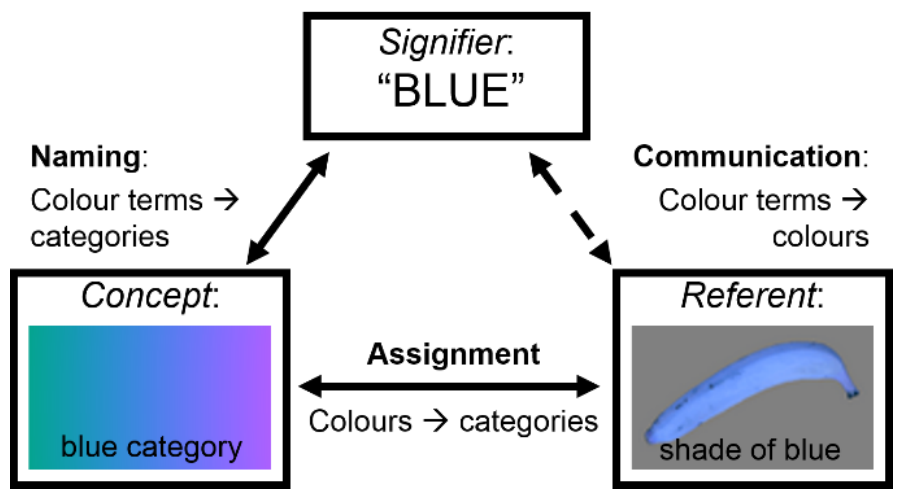

Figure 1. Colour perception and categorization. (a) Maximally saturated Munsell colour chips rendered under a white illumination based on the Finnish spectral database (Kohonen, Parkkinen, \& Jaaskelainen, 2006). Chips are arranged by hue along the $\mathrm{x}$ - and by lightness along the $\mathrm{y}$-axis (cf. insets). (b) Colour categories measured for the chips in (a) by Olkkonen, Witzel, Hansen, and Gegenfurtner (2010). (c) Triangle of reference illustrated for colour categories. Note that displayed colours can vary depending on print and display devices and are for approximate illustration only.

\section{Categorical perception}

Categorical perception has been the major approach to establish a link between perception and categorization and has become a mainstay in the perceptual and cognitive literature (e.g. Collins \& Olson, 2014; E. B. Goldstein, 2014; Goldstone \& Hendrickson, 2010; Harris, 2014; Harnad, 2017). According to the idea of categorical perception (cf. Figure 2.a), differences between stimuli (e.g. between bluish and purplish colours) are perceived as larger when colours straddle a category boundary (e.g. between the categories blue and purple) than when they belong to the same category (e.g. to purple) (Bornstein, 1987; Harnad, 1987). This idea has been originally introduced in research on phoneme perception. Discrimination of sounds along the transitions between the phonemes $[\mathrm{b}],[\mathrm{g}]$, and [d] is more accurate when these sounds cross the boundaries between $[\mathrm{b}],[\mathrm{g}]$, and [d], which may be considered as phoneme categories (Liberman, Harris, Hoffman, \& Griffith, 1957). Besides phonemes, colour has become the other major example for categorical perception (Bornstein, 1987; Harnad, 1987; Collins \& Olson, 2014; Harnad, 2017). The idea that colour perception is categorical has been so widely accepted that it is found in current text books (e.g. E. B. Goldstein, 2014, p. 322; Harris, 2014, p. 401), and scientific dissemination to the broader public (e.g. Deutscher, 2011; Robinson, 2011). The stripes of the rainbow have been presented as an obvious everyday-life example of categorical colour perception, in which colours are perceived as distinct stripes even though the spectrum of the 


\section{Misconceptions about colour categories}

rainbow is continuous (e.g. Collins \& Olson, 2014; Gaissert, Waterkamp, Fleming, \& Bulthoff, 2012; E. B. Goldstein, 2014, p. 322; Goldstone \& Hendrickson, 2010; Harris, 2014, p. 401; Livingston, Andrews, \& Harnad, 1998;

Özgen, 2004). To explain categorical perception, it has been reported that colour perception is "warped" such that differences between colours that belong in different categories are accentuated, and differences between colours that fall into the same category are deemphasized (p. 69, Goldstone \& Hendrickson, 2010; see also e.g. Drivonikou et al., 2007; Franklin, 2009; Franklin \& Davies, 2004; Goldstone 1994a, 1995; Özgen, 2004). Some other scientific studies present colour perception as categorical because they use the term "categorical perception" synonymously with colour categorization (e.g. Shinoda, Uchikawa, \& Ikeda, 1993; H. Uchikawa, Uchikawa, \& Boynton, 1989; K. Uchikawa, 2014; K. Uchikawa \& Boynton, 1987; Yang, Kanazawa, \& Yamaguchi, 2013; Zollinger, 1988).

From such sources, one might get the impression that colour perception per se is inherently categorical (see in particular Livingston, Andrews, \& Harnad, 1998; Goldstein, 2014, p. 322; Goldstone \& Hendrickson, 2009; Harnad, 2017).

However, such a conclusion confuses the concept of categorical perception and misrepresents empirical evidence (see also Ocelák, 2016).

\section{The core problem}

\section{The meaning of "categorical"}

The concept of categorical colour perception does not necessarily mean that colour perception is completely categorical in the sense that we are unable to distinguish between colours from the same category (see also Franklin \& Davies, 2004). Instead, colour perception allows for seeing gradual changes in colour, within and across categories, such as in the coloured background of Figure 2.a. This implies that colour perception is continuous and this is also the basis for representing colour differences metrically in three-dimensional colour spaces. Categorical perception should not be confused with colour categorization. The fact that observers assign colours to categories that correspond to colour terms is not the same as the idea that colours are perceived in a categorical way. Given the continuous character of colour perception and the difference between perception and categorization, a sensible approach to categorical colour perception calls for 


\section{Misconceptions about colour categories}

categorical patterns that is modulations in colour perception that are specific to colour categories (Witzel \& Gegenfurtner, 2013, 2014, 2015, 2016, 2018, in press). For example, colours might change gradually across categories, but the colour differences could be more obvious at the category border than within the category (cf. Figure 2.a). Such categorical patterns would link linguistic categories that correspond to colour terms to continuous colour perception.

\section{Contradictory empirical evidence}

However, evidence for categorical perception has been contradictory. It has been observed that colour vision is modulated by differences in colour categorization across languages (e.g. Roberson, Davidoff, Davies, \& Shapiro, 2005; Roberson, Davies, \& Davidoff, 2000; Roberson, Pak, \& Hanley, 2008; Thierry, Athanasopoulos, Wiggett, Dering, \& Kuipers, 2009; Winawer et al., 2007), by language-specific interference tasks (Gilbert, Regier, Kay, \& Ivry, 2006; Pilling, Wiggett, Özgen, \& Davies, 2003; Roberson \& Davidoff, 2000; Winawer et al., 2007), by learning new categories (Goldstone, 1994b, 1995; Özgen, 2004; Özgen \& Davies, 2002; Zhou et al., 2010), and by the lateralization of language in the brain (Drivonikou et al., 2007; Gilbert et al., 2006; Roberson et al., 2008; Zhou et al., 2010). These observations suggested effects of categories on perception (category effects), implying that linguistic colour categorisation shapes colour perception.

Other studies claimed the inverse, namely that linguistic categories result from the categorical nature of colour perception. They found that colour vision itself is inherently categorical (Regier, Kay, \& Khetarpal, 2007), and that colour categories already exist in infancy before language acquisition (Bornstein, Kessen, \& Weiskopf, 1976; Franklin, Clifford, Williamson, \& Davies, 2005; Franklin \& Davies, 2004; Franklin, Drivonikou, Bevis, et al., 2008; Franklin, Drivonikou, Clifford, et al., 2008; Franklin, Pilling, \& Davies, 2005; Ozturk, Shayan, Liszkowski, \& Majid, 2013; Skelton, Catchpole, Abbott, Bosten, \& Franklin, 2017). Still other findings did not find categorical patterns in colour perception and contradicted the idea of categorical perception (Bachy et al., 2012; A. M. Brown, Lindsey, \& Guckes, 2011; Cropper, Kvansakul, \& Little, 2013; Davidoff, Goldstein, Tharp, Wakui, \& Fagot, 2012; Gerhardstein, Renner, \& Rovee-Collier, 1999; Grandison et al., 2016; Kay \& Kempton, 1984; Lindsey et 


\section{Misconceptions about colour categories}

al., 2010; Roberson, Hanley, \& Pak, 2009; Webster \& Kay, 2012; Witzel \& Gegenfurtner, 2011, 2013). How is it possible that some studies observe effects of categories on perception, other studies find that perception determines categories, and still others do not find any evidence for categorical perception?

The problem of stimulus control

A fundamental problem with comparing and evaluating the results of those studies is the question of how they controlled the stimuli (i.e. the light or surfaces used to measure the perception of colour differences) in order to reveal a categorical pattern in the perception of colour differences (Kay and Kempton, 1984; Webster \& Kay, 2012; Witzel \& Gegenfurtner, 2013, 2015, 2016, 2018; for review see Witzel \& Gegenfurtner, 2014; in press). To establish empirical evidence for categorical perception, the continuous, non-categorical differences between colours must be controlled to make colours within and across categories comparable and to reveal a categorical pattern. For example, the stimulus difference between a blue and a purple colour should be comparable to the difference between two blue colours (cf. Figure 2.a). If stimulus differences are not equivalent, then perceived differences will be affected by the differences between stimuli. Resulting modulations of perceived colour differences are prone to confuse effects of stimulus control with effects of colour categorization, hence making it difficult to disentangle which effects are simply due to continuous perception and which are specific to linguistic categories.

For this reason, tests of categorical perception rely on a comparison between (1) a category-probing metric, i.e. a quantitative measure that shows the categorical character of perception in contrast to the continuous variation of the stimulus, (2) a reference metric, i.e. a quantitative measure that defines stimuli in a continuous way and allows for the specification of comparable stimulus differences (Witzel \& Gegenfurtner, 2013, 2014, 2015, 2016, 2018, in press). Note that any measure of perception cannot directly assess subjective perception itself (i.e. the percept). It measures responses in a task that is assumed to reflect perception. Different tasks are available that presumably reflect different aspects of perception. As a result, the specification of the category-probing and the reference metric is the heart of the concept of categorical perception because it determines what 


\section{Misconceptions about colour categories}

exactly is meant by categorical perception. Let's first consider what reference metric could be used to make stimulus differences equivalent.

\section{Classical approaches}

\section{Physical reference metrics}

The original and still widespread approach has been to define categorical perception as a categorical pattern in perception when perception is compared to a continuous physical measure (Bornstein, 1987; Bornstein \& Korda, 1984; Harnad, 1987, Livingston, Andrews, \& Harnad, 1998; see also Goldstein, 2014, p. 322; Goldstone \& Hendrickson, 2009; Harris, 2014, p. 401). Consider Figure 3, which illustrates schematically the different levels of color processing. The physical property that corresponds to colour perception is the wavelength composition (spectra) of the light that impinges on the receptors in the retina (impinging spectra in Figure 3). The first studies on categorical colour perception have compared colour perception to wavelength differences between spectral colors that is colours that are defined by one single wavelength (Bornstein, Kessen, \& Weiskopf, 1976; see also Holtsmark \& Valberg,1969).

One problem with this approach is that unambiguous wavelength differences can only be determined for spectral colours. Such colours occur when light is refracted into monochromatic light by a prism. However, apart from this particular case, almost all colours in our environment are the result of wavelength spectra, i.e. a range of different wavelengths. Even the famous example of the rainbow is not an instance of spectral colours (Lee, 1991; Smithson et al., 2014). The wavelength composition of the light coming from a rainbow is the result of light refraction at the surface of a multitude of tiny water droplets. The result is a complex interference between monochromatic lights, the perception of which is not directly comparable to the perception of single monochromatic lights. It has been claimed that the stripes perceived in a rainbow might be the result of categorical perception (e.g. Collins \& Olson, 2014; Goldstone \& Hendrickson, 2010; Hardin, 1988). If so, we might need to consider this to be a special case that is not necessarily representative for all visible colours.

Another problem with using wavelength differences for stimulus definition is the fact that the sensitivity of the cones to spectral light is non-linear (cf. Figure 2.a), and hence it is not surprising that the sensitivity to wavelength differences is non- 


\section{Misconceptions about colour categories}

linear, too (Holtsmark \& Valberg,1969; Wright \& Pitt, 1934; see also Bachy, Dias, Alleysson, \& Bonnardel, 2012; Mullen \& Kulikowski, 1990). It might be claimed that these non-linear transitions across wavelengths correspond to a kind of perceptual categories. However, if so these categories do not correspond to the categories of Basic Colour Terms (e.g. Beare, 1963; Holtsmark \& Valberg,1969; Wright \& Pitt, 1934; see also Bachy, Dias, Alleysson, \& Bonnardel, 2012). In sum, using wavelength differences it is difficult to disentangle the non-linear effect of cone sensitivities and categorical perception, and to relate those results to everyday colour perception, which mainly involves non-spectral lights.

Dominant wavelength, another measure used in early studies on categorical colour perception (Bornstein \& Korda, 1984; Goldstone, 1995; see also Goldstone \& Hendrickson, 2009), might seem to partially solve the above problems and to provide a physical measure for non-spectral colours. Dominant wavelength corresponds to the projection of the chromaticity coordinates of a colour (more precisely a colored light) on the spectral locus, i.e. the horseshoe-shaped curve that spectral colors form in the chromaticity diagram. The chromaticity diagram basically represents the sensory signal at the first-stage of colour processing in the retina. Hence, it is rather a sensory than physical measure (cf. Figure 3). More importantly, the perception of differences in the chromaticity diagram strongly varies across the visible gamut in a way that is unrelated to colour categories. This can be seen from the variation of discrimination thresholds in chromaticity space (Macadam, 1942; Boynton \& Kambe, 1980). In addition, dominant wavelengths as well as the chromaticity diagram strongly depend on the state of adaptation, i.e. the chromaticity of the light that is perceived as white (e.g. Krauskopf \& Gegenfurtner, 1992). As a result, the nonlinearities observed across the chromaticity diagram vary depending on the observer's state of adaption, implying that the chromaticity diagram might not be a reliable reference for evaluating categorical patterns.

\section{Perceptual reference metrics}

The strong variation of perceived colour differences due to factors other than colour categories may distort empirical evidence for categorical colour perception. On the one hand, spurious categorical patterns due to stimulus sampling may result when stimuli are sampled sparsely and larger differences accidentally 


\section{Misconceptions about colour categories}

happen to occur between colours that cross the category boundary. On the other hand, strong variation of colour differences may cover potential categorical patterns.

A solution to that problem is to compare measures of categorical perception to a continuous stimulus dimension that is defined by a non-categorical perceptual instead of a physical reference metric (Kay \& Kempton, 1984). In line with this logic, studies used stimuli that aimed at equating "perceptual distances", i.e. colour differences according to a "perceptual metric" (cf. in Drivonikou et al., 2007; Franklin \& Davies, 2004; Franklin \& Davies, 2005; Franklin, Drivonikou, Clifford, et al., 2008; Özgen \& Davies, 2002; Özgen, 2004; Pilling et al., 2003; "discrimination distance" in Kay \& Kempton, 1984). For this, studies on categorical perception (Bornstein \& Korda, 1984; Kay \& Kempton, 1984) started to use Munsell chips to equate perceptual distances, as suggested by studies on the perceptual spacing of Munsell Chips (Newhall, Nickerson, \& Judd, 1943) and introduced to the study of color naming earlier on (Brown \& Lenneberg, 1954; Lenneberg, 1953). Almost all subsequent studies on categorical perception followed this approach and equated perceptual distances according to the Munsell System. A few also used CIELUV and CIELAB colour space for this purpose (e.g. Pilling et al., 2003; Roberson, Hanley, \& Pak, 2009). However, the Munsell System, CIELUV and CIELAB space are all designed to approximate perceived differences (e.g. Fairchild, 2013; Hunt \& Pointer, 2011). There is a circularity in the logic of this approach: If categories affect colour perception, then these effects should be part of those measures of colour differences. In other words, it is logically contradictory to test for variation of colour perception depending on colour categories when claiming at the same time that colour perception does not vary due to stimulus control (e.g. Bimler, 2009; A. M. Brown et al., 2011; Firestone \& Scholl, 2016; Lindsey et al., 2010; Ocelák, 2016; Webster \& Kay, 2012; Winawer \& Witthoft, 2014; Witzel \& Gegenfurtner, 2013, 2014, 2015, 2016).

Another problem with this approach to stimulus control is that existing models of perceptual colour spaces are only coarsely approximate in their control of perceived colour differences across colour space - that is the reason why there are different approaches to model colour perception through colour spaces (e.g. 


\section{Misconceptions about colour categories}

Fairchild, 2013; Hunt \& Pointer, 2011). Depending on where in colour space categorical effects on perception are probed, different results may occur due to the imprecisions of stimulus control. Earlier studies on categorical colour perception focused on only a few categories that were considered to be representative. Most of them used Munsell chips at the green-blue boundary (for discussion see e.g. Ocelák, 2016; Witzel \& Gegenfurtner, 2013, 2014, 2015, 2016). Due to the inhomogeneity of those colour spaces, patterns found for one stimulus set may not be representative for category effects in general. If supposed category effects on colour perception appear despite the supposed control of colour perception, there is either a fundamental logical contradiction or an insufficient control of stimuli (A. M. Brown et al., 2011; Lindsey et al., 2010; Witzel \& Gegenfurtner, 2014, 2015, 2016). This may explain why many of the effects observed in previous studies disappeared when using different stimulus samples (Bachy, Dias, Alleysson, \& Bonnardel, 2012; A. M. Brown, Lindsey, \& Guckes, 2011; Cropper, Kvansakul, \& Little, 2013; Davidoff, Goldstein, Tharp, Wakui, \& Fagot, 2012; Gerhardstein, Renner, \& Rovee-Collier, 1999; Grandison et al., 2016; Lindsey et al., 2010; Roberson, Hanley, \& Pak, 2009; M. A. Webster \& Kay, 2012; Witzel, Cinotti, \& O'Regan, 2015; Witzel \& Gegenfurtner, 2011, 2013, 2016). Nevertheless, the modulation of category effects through language-specific categories, through language-specific interference tasks and through newly learned categories produces category effects for the same stimuli under one, but not under another condition. These interaction effects cannot be explained by the lack of stimulus control. So, where do the observed category effects come from? a.)

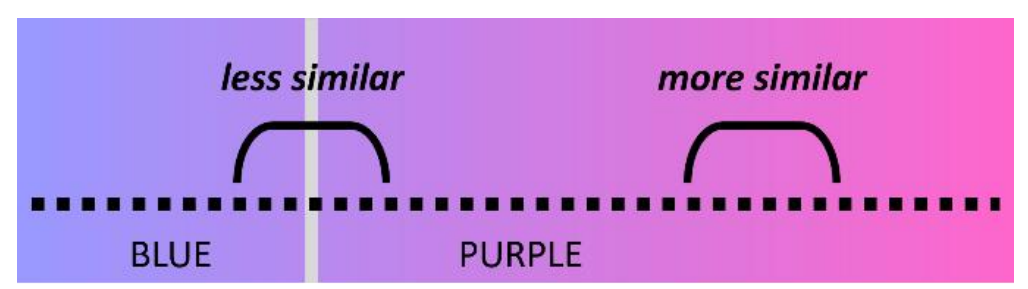

b.) 


\section{Misconceptions about colour categories}

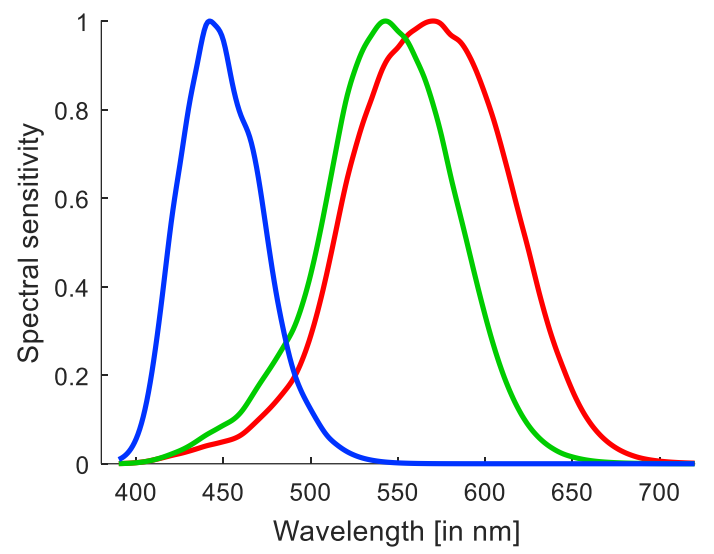

Figure 2. Categorical perception. (a) Basic idea of categorical perception. Grey vertical line indicates category boundary between blue and purple; "similar" refers to perceived difference; and the dotted black line indicates a continuous measure of reference (see text for discussion. (b) Cone sensitivities (Stockman \& Sharpe, 2000). Displayed colours are for approximate illustration only.

\section{Different levels of processing}

To understand where those category effects come from, it is useful to distinguish different aspects of colour perception (see already Kay \& Kempton, 1984) and we can test which aspects of colour perception are related to colour categorization and which are not. In this logic, tests of categorical perception compare (1) a measure of colour perception that is void of categorical patterns (i.e. the perceptual reference) and (2) another measure of colour perception that tests for categorical patterns (i.e. the category-probing measure).

In particular, we can compare measures of colour perception at different levels of perceptual processing (A. M. Brown et al., 2011; Cropper et al., 2013; Lindsey et al., 2010; M. A. Webster \& Kay, 2012; Witzel \& Gegenfurtner, 2013, 2014, 2015, 2016, 2018, in press). In this approach, a continuous measure at a low level of visual processing is used as the perceptual reference to control colour stimuli. To test for categorical patterns, a task is used that builds on the low-level information, but may potentially involve effects from higher levels of processing. If categorical patterns occur, categorical perception may be attributed to a particular level of perceptual colour processing depending on which perceptual reference and which category-probing task have been used (Witzel \& Gegenfurtner, 2013, 2014, 2015, 2016, 2018, in press).

\section{Sensory stages}

A first step into this direction consists in defining the perceptual reference by lowlevel sensory measures of colour perception (Figure 3). The first stage of colour 


\section{Misconceptions about colour categories}

perception is the excitation of the photoreceptors in the retina (e.g. Gegenfurtner \& Kiper, 2003). The light that reaches the retina is encoded by the cones, which are the photoreceptors involved in colour vision. There are three types of cones that differ in their sensitivity to long- (L-cones), medium- (M-cones), and shortwavelength (S-cones) light (cf. Figure 2.b). The second stage of colour processing contrasts the cone-excitations, producing the cone-opponent mechanisms that could be shown in the retinal ganglion cells and the lateral geniculate nucleus (LGN) in the thalamus (Derrington et al., 1984). The resulting second-stage mechanisms of colour perception are formed by an absolute lightness dimension (more precisely called "luminance") that adds the excitation of L- and M-cones $(\mathrm{L}+\mathrm{M})$, the contrast between $\mathrm{L}$ and $\mathrm{M}$ cones $(\mathrm{L}-\mathrm{M})$, and the contrast between Scones and luminance $(\mathrm{S}-[\mathrm{L}+\mathrm{M}])$. These mechanisms define the fundamental dimensions, or cardinal axes, of colour discrimination, and the colour signal at the second stage of colour processing can be modelled by the Derrington-KrauskopfLennie (or DKL-) colour space (Derrington, Krauskopf, \& Lennie, 1984; Krauskopf, Williams, \& Heeley, 1982). Due to adaptation and following Weber's Law, the sensitivity of those mechanisms to hue differences is highest when colours fall along one of the cardinal axes (Krauskopf et al., 1982; Krauskopf \& Gegenfurtner, 1992; Giesel, Hansen, \& Gegenfurtner, 2009). Thus, category boundaries should coincide with the cardinal axes L-M and S-(L+M) if categorical perception (for linguistic colour categories) was inherent to the sensitivity of the second-stage mechanisms. This, however, is not the case. For example, while the negative pole of the L-M dimension coincides with the greenblue boundary, the positive pole of the $S-(\mathrm{L}+\mathrm{M})$ dimensions corresponds to the most typical purple rather than to a category boundary (e.g. Figure 1 in Malkoc, Kay, \& Webster, 2005; Figure 9 in Witzel \& Gegenfurtner, 2013; Figure 1 in Witzel \& Gegenfurtner, 2018).

\section{Categorical sensitivity}

The next step along the hierarchy of colour processing consists of comparing the sensitivity to colour differences to the sensory signal provided by the second-stage mechanisms (Figure 3). The sensitivity to colour differences is the basic ability to discriminate colours. This sensitivity is partly determined by second stage mechanisms (Krauskopf et al., 1982; Krauskopf \& Gegenfurtner, 1992), but 


\section{Misconceptions about colour categories}

cortical higher-order mechanisms may play an important role in the shaping sensitivity, too (Eskew, 2009; Giesel, Hansen, \& Gegenfurtner, 2009; Hansen \& Gegenfurtner, 2013). Sensitivity can be empirically measured through performance in colour discrimination tasks and most notably through discrimination thresholds. It turned out that colour discrimination and discrimination thresholds are shaped by second-stage mechanisms, but not by colour categories (A. M. Brown et al., 2011; Lindsey et al., 2010; Witzel \& Gegenfurtner, 2013, 2014, 2016, 2018). Since the green-blue boundary coincides with these mechanisms (e.g. Figure 1 in Malkoc et al., 2005; Figure 9 in Witzel \& Gegenfurtner, 2013; Figure 1 in Witzel \& Gegenfurtner, 2018), high sensitivity along these mechanisms potentially explains the higher discrimination performance at the green-blue boundary that was attributed to categorical perception in previous studies (for discussion see also Witzel \& Gegenfurtner, 2013, 2014, 2015, 2016, 2018, in press).

\section{Categorical facilitation}

As the next step, we can use sensitivity to colour differences as our perceptual reference, and test whether there are category effects at higher, more cognitive levels of processing. When carefully controlling sensitivity to colour differences, effects of linguistic colour categories could be observed (Witzel, 2012; Witzel \& Gegenfurtner, 2014, 2015, 2016). These effects are thus "high-level effects", i.e. they cannot be attributed to fundamental low-level sensory aspects of colour vision but rather to attention and subjective evaluation (Roberson et al., 2009; Witzel \& Gegenfurtner, 2014, 2015, 2016). It may be debated whether such effects should still be considered as effects on perception in the strict sense; but if we define perception in a broad sense as direct responses to a present stimulus, these effects could still be called categorical perception. However, to distinguish these effects from other forms of categorical perception we proposed to call this phenomenon categorical facilitation as a special kind of categorical perception (Witzel \& Gegenfurtner, 2014, 2015, 2016).

The idea of categorical facilitation is in line with previous findings that show category effects for language specific categories, for newly learned categories, and its dependence on verbal interference: If categorical facilitation is due to the 


\section{Misconceptions about colour categories}

observers focusing their attention on the differences between linguistic categories, this explains why category effects on perception are specific to languages and may appear for new categorical distinctions. Verbal interference might modulate categorical facilitation by deviating attention from the verbal distinction. Most recently, a Bayesian model has been introduced to account for top-down effects of categories on colour perception (Cibelli, Xu, Austerweil, Griffiths, \& Regier, 2016; Holmes, Moty, \& Regier, 2017) and memory (Bae, Olkkonen, Allred, \& Flombaum, 2015). Category knowledge is considered as a prior and its integration with perceptual information results in category effects. This model is in line with the idea of categorical facilitation. In addition, it links category effects to other kinds of top-down effects on colour perception, for which similar Bayesian models have been proposed (e.g. effects of short-term memory on colour appearance: Olkkonen \& Allred, 2014; Olkkonen, McCarthy, \& Allred, 2014; memory colour effects: Witzel, Olkkonen, \& Gegenfurtner, 2016; Witzel, Olkkonen, \& Gegenfurtner, 2018; for discussion see Witzel \& Gegenfurtner, in press).

\section{Open questions}

Although there is converging evidence against the idea that colour perception at the early stage of processing is inherently categorical, it still remains unclear where the high-level effects of categorical facilitation come from. It may be speculated that categorical facilitation is due to attention; but this has not yet been proven. A major challenge for further investigating the origin of categorical facilitation is that our knowledge about high-level mechanisms of colour perception is fragmented and incomplete, and we are far from being able to precisely model every aspect of subjective colour appearance (cf. Figure 3; for discussion, see e.g. Cropper et al., 2013; Cropper \& Wuerger, 2005; Gegenfurtner \& Kiper, 2003; Witzel \& Gegenfurtner, in press; Wuerger, Maloney, \& Krauskopf, 1995). For example, response times in speeded, suprathreshold discrimination, such as in typical categorical perception tasks, are affected by salience, which is determined by the difference of the colours from the adaptating background (e.g. Cropper et al., 2013). Yet it is unclear how to fully control for 


\section{Misconceptions about colour categories}

the effects of salience and saturation (cf. Witzel, in press; see also section 3 on focal colours).

In addition, evidence for the lateralisation of category effects to the right visual field is contradictory: Several studies could not reproduce the lateralisation of category effects (A. M. Brown et al., 2011; Lu, Hodges, Zhang, \& Wang, 2012; Suegami, Aminihajibashi, \& Laeng, 2014; Webster \& Kay, 2012; Witzel \& Gegenfurtner, 2011, 2015, 2016). In particular, categorical facilitation measured with carefully controlled stimuli did not show any lateralization (Witzel \& Gegenfurtner, 2015, 2016). There is some evidence that lateralized categorization may be produced independent of linguistic categories, suggesting that the lateralization is due to differences in attention to the visual fields (Alvarez, Clifford, Holmes, \& Franklin, 2012). Others have argued that the lateralization of category effects depends on the time course of perceptual processing (Roberson et al. 2008; Constable \& Becker 2017). Nevertheless, it remains an open question why lateralisation was found in some but not in other studies.

Finally, since infants do not yet have language, evidence for infant colour categories are a major challenge for language based explanations of categorical colour perception (Bornstein et al., 1976; Franklin \& Davies, 2004; Franklin, Pilling, et al., 2005; Skelton et al., 2017). One possibility is that linguistic categories reflect regularities in the visual environment, which guide the attention of preverbal infants to patterns in the environment (Franklin, Wright, \& Davies, 2009). Alternatively, infants could also learn to pay attention to linguistic differences between categories through social interaction, in particular through joint attention (Moore \& Durham, 1995). In contrast, a recent study suggested that the boundaries of infants colour categories align with the cardinal axes of the second-stage mechanisms of colour perception (Skelton et al., 2017). This finding contradicts the observation that adult category boundaries do not align with those sensory mechanisms (Malkoc et al., 2005; Witzel \& Gegenfurtner, 2013, 2015, 2016,2018 ), and raises the question of how infant colour categories are related to linguistic categorization in adults (Ocelak, 2016). The particular challenge with infant data is that it is indirect and thus its precise interpretation may be difficult. In particular, it is difficult to distinguish whether behavioural measurements in infants, such as novelty preferences, measure effects of attention, preference, 


\section{Misconceptions about colour categories}

discrimination, categorisation, or a combination of these (Brown \& Lindsey, 2013; Franklin, 2009; Roberson, \& Hanley, 2009).

\section{Summary}

In sum, tests of categorical perception rely on the comparison of two measures, one that is assumed to be continuous (reference metric) and another that is expected to show categorical patterns (category-probing measure). Tests of categorical perception depend on how these two measures are determined and conclusions about categorical perception depend on assumptions about how those measures relate to perception. State of the art research undermines the idea that colour perception (in adults) is inherently categorical. Instead, there is converging evidence for categorical facilitation, i.e. top-down effects of linguistic categorisation on perception, which is probably due to attention. These effects are not reliably lateralized to the right visual field. It remains an open question where categorical facilitation comes from, whether and under which conditions it is lateralised, and how it is related to infant colour categories.

\section{Physical characteristics}

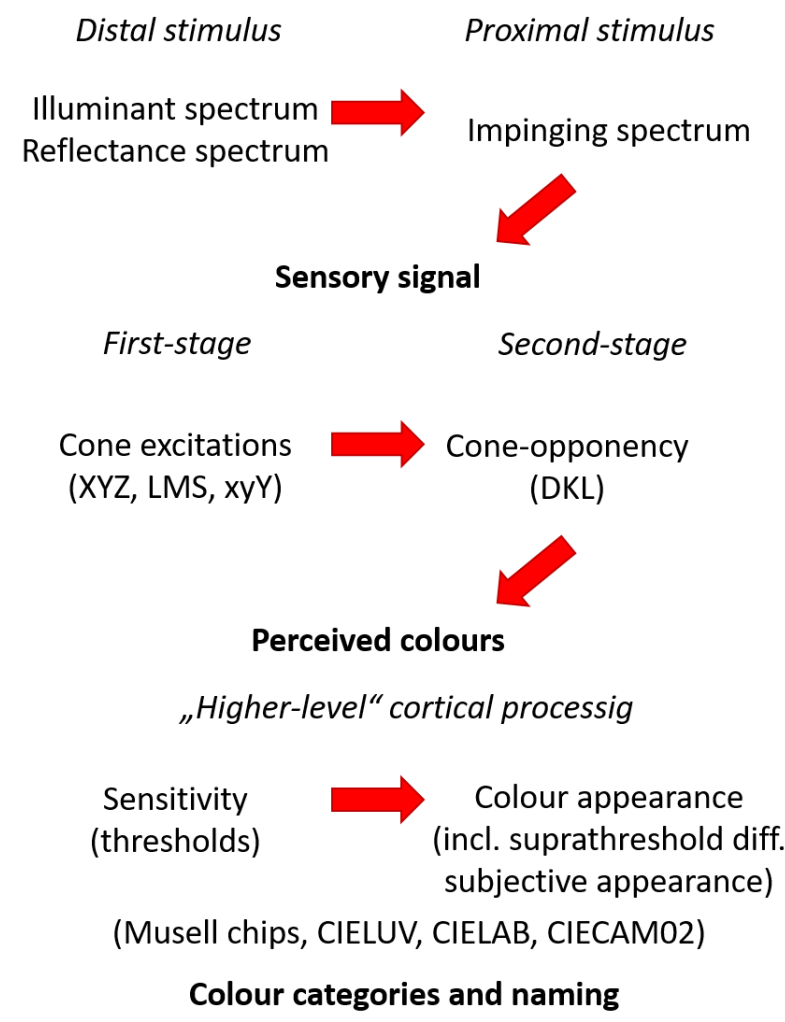

Figure 3. Schematic overview of the stages of colour processing. The distal stimulus are physical properties (spectra) of surfaces and lights; the proximal stimulus are the properties of the light that 


\section{Misconceptions about colour categories}

impinges on the retina in the eye. First-stage mechanisms relate to the excitation of the three photoreceptors in the retina, the cones, and can be modelled using cone sensitivities (LMS; see also Figure 2.b) and colour matching functions (XYZ, xyY). Second-stage mechanisms compare the signal from the different types of cones and are implemented in the retinal ganglion cells and the LGN of the thalamus (see e.g. Gegenfurtner \& Kiper, 2003). The nature of higher-level mechanisms that determine sensitivity and discrimination thresholds is still under debate (e.g. Eskew, 2009; Hansen \& Gegenfurtner, 2013) and several models of colour appearance have been proposed (in particular Munsell chips, CIELUV, CIELAB, and CIECAM02; see e.g. Fairchild, 2013; Hunt \& Pointer, 2011). This makes it difficult to determine how colour categorization and naming are related to color perception.

\section{Basic Color Terms}

It is crucial to define and identify colour categories in order to study colour categorisation and the relationship between perception and language. For example, we need to know the boundaries between categories to investigate categorical perception; or we need to identify categories in different languages to study the variation of colour categorisation across languages.

It might seem intuitive that there are only a few colour terms, such as red, orange, and brown, that clearly define linguistic colour categories. Yet, there may be a large range of candidate color categories if we consider all color terms that exist in a language. For example, American English includes colour terms such as peach, teal, lavender, maroon, tan, gold, turquoise, burgundy, aqua, violet, salmon, magenta, olive, fuchsia, lime, periwinkle, lilac, mustard, beige and many more (Lindsey and Brown, 2014; see also Mylonas \& MacDonald, 2016; Paramei, Griber, \& Mylonas, 2018). The use of those colour terms depends on the speakers and the context. In fact, specialists within a language community, such as painters or graphic designers, may develop a broader range of labels for colours to enable a more specific communication in their professional context. An extreme example is the colour codes in standardized colour atlases, such as Pantone, NCS, or Munsell Chips. These codes label almost every discriminable colour along the three dimensions of colour perception and barely involve grouping colours in categories.

However, the codes of colour atlases are an example of colour labels that are tailor-made for communication in specific professional contexts and are not used to communicate colours in everyday life. Furthermore, many of the above colour terms, such as periwinkle and aqua, are used very rarely and only by a few 


\section{Misconceptions about colour categories}

speakers. So, not all labels for colours are automatically colour terms, and not all colour terms refer to colour categories that are equally important for communicating colours in a language community. But how can we identify the colour terms and categories that are appropriate to study colour categorisation?

\section{The original notion of Basic Colour Terms}

In their ground-breaking study Berlin and Kay (1991 [1969]) introduced the notion of basic colour terms, which allowed them to compare colour categories across fundamentally different languages. Basic colour terms were defined by four criteria. First, they are monolexemic, i.e. they consist of only one lexeme (meaningful word). "Dark blue" is a counter-example because it is composed of two words (dark and blue). Second, Basic colour terms are used with high consistency by each speaker and with high consensus across different speakers to describe the same colours. A counter-example may be turquoise in German because some observers use it exclusively for light, others for dark and still others for all mixtures of green and blue (Witzel, 2012, p. 250ff; Figure S3 in Witzel \& Gegenfurtner, 2011). Third, basic colour terms are not restricted to a narrow class of objects (context independence). For example, blond contradicts this criterion because it is only applied to hair. Finally, basic colour terms should be hypernyms, i.e. they should describe colour categories at the highest level of generality and subsume all non-basic colour categories. For example, the non-basic colour word crimson is part of the red category. Apart of these primary criteria, there were secondary criteria, which should be applied in case that the primary criteria could not unambiguously establish whether a colour term is basic or not (Berlin and Kay (1991 [1969], p.5-7). For example, the secondary criteria excluded foreign loan words and colour terms that consisted of object names (homonymy), but only if they did not satisfy all primary criteria. According to these criteria, there are eleven English basic colour terms: black, grey, white, pink, red, orange, brown, yellow, green, blue, and purple.

The idea of basic colour terms has been supported by empirical measures that empirically determined the "basicness" of colour terms, i.e. the degree to which colour terms may be considered as "basic". They showed that colour naming with the conceptually defined eleven basic colour terms in English is faster and intraand inter-individually more consistent than naming with other, non-basic colour 


\section{Misconceptions about colour categories}

terms (Boynton \& Olson, 1990; Guest \& Van Laar, 2000; Sturges \& Whitfield, 1997).

The criteria for basic colour terms have been used to establish the number of basic colour terms for a large range of languages across the world. Apart from the original study of Berlin and Kay (1991 [1969]), the World Colour Survey may be considered as the most prominent example, which identified the basic colour terms in 110 non-industrialised languages (Kay, 2015; Kay, Berlin, Maffi, Merrifield, \& Cook, 2011; for overview see Kay, 2015; Kay \& Maffi, 1999; Kay, $\&$ Regier, 2003). The notion of basic colour terms is so widely established that it features in practically every general introduction to colour categorisation (e.g. Deutscher, 2011; Gage, 1999, p.79ff; Hansen, 2010; Kremers, Baraas, \& Marshall, 2016; MacLaury, Paramei, \& Dedrick, 2007, Paulsen, Uusküla, \& Brindle, 2016; Shevell, 2003) and it has been taken as the starting point for a large variety of studies related colour naming and categorisation (e.g. Baronchelli, Gong, Puglisi, \& Loreto, 2010; Loreto, Mukherjee, \& Tria, 2012; Steels \& Belpaeme, 2005). So, it may appear that basic colour terms are a clear-cut linguistic phenomenon that exists in every language and can be unambiguously identified using the original criteria.

\section{Criticism and updates}

However, the definition of basic colour terms has been strongly criticised (e.g. Biggam, 2012; Crawford, 1982; Dedrick, 1998; Durbin, 1972; Hickerson, 1971, 1980; Lucy, 1992, 1997a, 1997b; Lucy \& Shweder, 1979, 1988; Merrifield, 1971; Mills, 1984; Moss, 1989; Rosch Heider, 1972a; Saunders, 1995, 2000, 2007; Saunders \& van Brakel, 1997; Wescott, 1970). Although the criteria of basic colour terms intuitively seem to be quite appropriate to identify important colour terms in English and languages with a similar structure; we do not know what is an important and fundamental colour term in other languages. So, there is no guaranty that the conceptual criteria of basic colour terms are equally valid for every existing language. It should be noted that Berlin and Kay (1991 [1969], p.57) introduced those conceptual criteria for basic colour terms not as rigorous laws, but as guidelines, as can be seen from the auxiliary character of the secondary criteria (see also Biggam, 2012, p.22). Nevertheless, these criteria have been 


\section{Misconceptions about colour categories}

applied rigorously to establish the nominal number of basic colour terms for languages across the world. The uncritical application of the conceptual criteria for basic colour terms may preclude the identification of otherwise important and quite fundamental colour terms just because they disagree with those particular criteria of basicness.

\section{Colour terms and objects}

One reason for this may be the criterion of context independence and the secondary criterion of homonymy because a rigorous dissociation between object names and colour terms is not always possible. Many non-industrialised language communities use colour terms that refer to objects (Brown et al., 2016; Groh, 2016; Haynie \& Bowern, 2016; Levinson, 2000; Saunders \& van Brakel, 1997). Such colour terms may be fundamental to the colour vocabulary of a language. Yet, they may not fit the classical definition of basic colour terms because they are homonymous with objects and their usage may be partly bound to a particular context or range of objects (Levinson, 2000).

A particularly important example for this problem may be the case of the Dugum Dani, an indigenous tribe in West Papua (former New Guinea, now Indonesia). Based on an ethnographic study from 1965 (cf. Heider, 1971), Berlin and Kay (1991 [1969]) and Rosch Heider (1971, 1972b) reported the Dugum Dani to have only two colour terms, mili and mola (Heider, 1971). Since then the Dugum Dani are widely considered to be the only known example of a language that has only two colour terms (e.g. Kay, 2015; Roberson, Davies, \& Davidoff, 2000; see also the discussion in Saunders \& van Brakel, 1997). However, a recent study claimed that the Dani have colour terms for almost all colours that correspond to the prototypes of English basic colour terms, but most of those colour terms were object names (Groh, 2016). Berlin and Kay (1991 [1969], p.46-47) and Rosch Heider (1972a) had already discussed additional Dani colour terms, including gut for white and gareka for green, which were also found in that recent study (Groh, 2016). However, they considered those colour terms to contradict the criterion of context independence and homonymy. It is difficult to compare findings about Dani colour terms across those studies because they are separated by about 60 years. It is possible that colour terms changed during that time, in particular 


\section{Misconceptions about colour categories}

because the Dani were subject to contact with industrialized culture (e.g. through ethnographic film production, Gilberthorpe, 2017). Nevertheless, the example of the Dani illustrates that the relationship between colour terms and objects is decisive for research on basic colour terms (see also section "Universalism vs. Relativism" for the role of Dani colour terms in the evolutionary sequence). Furthermore, the relationship between colour terms and objects may also be found in the history of many Indo-European languages and industrialised societies, such as English and German (Conklin, 1973; Witzel, 2012). For example, the English colour term red (and rot in German) can be traced back to the Proto-IndoEuropean "reudh" for red ochre and copper and is related to the Sanskrit word „rudhira“ for blood (Alexander \& Kay, 2014; Jones, 2013). “Green” (and “grün” in German) have their roots in the Germanic word "ghro" that refers to growing and flourishing (Jones, 2013; Welsch \& Liebmann, 2004, p. 64); and "orange" originates in the Sanskrit word narangah for orange tree (Jones, 2013).

The reason why industrialised societies "forgot" the original links to objects might be that they developed technical means to isolate colour pigments and manipulate colours independently of objects (McNeill, 1972). It has been proposed that colour terms might have emerged out of non-colour expressions (Levinson, 2000; see also Rosch Heider,1972a). If this is so, the relationship between colour terms and non-colour expressions, including objects, may be blurry and a definition that a priori dissociates basic colour terms from non-colour context may be misleading. In particular, the criteria of context independence and homonymy were simply meant to distinguish colour terms from object names; but an uncritical application of these criteria may lead to underestimate the color vocabulary in languages that use object names to communicate about colours.

\section{Consistency and consensus}

Consistency within speakers and consensus across speakers is fundamental for successful communication. Yet, it has been observed that there can be systematic individual variation of colour term usage within a language community (A. M. Brown, Isse, \& Lindsey, 2016; Gibson et al., 2017; Kay et al., 2011, p.549; Kuriki et al., 2017; Lindsey \& Brown, 2009; Lindsey, Brown, Brainard, \& Apicella, 2015; Lindsey, Brown, Brainard, \& Apicella, 2016; Rosch Heider, 1972a; 


\section{Misconceptions about colour categories}

Webster \& Kay, 2007), including American English (Emery, Volbrecht, Peterzell, \& Webster, 2017; Lindsey \& Brown, 2014) and German (Olkkonen et al., 2010; Witzel \& Gegenfurtner, 2013; Witzel, Hansen, \& Gegenfurtner, 2008).

Related to the observation of individual differences in the usage of basic colour terms is the suggestion that there is not a clear-cut dichotomy between basic and non-basic colour terms. Instead, colour terms may have different degrees of "basicness" (A. M. Brown et al., 2016; Gibson et al., 2017; Lindsey \& Brown, 2009, 2014; Lindsey et al., 2015). For example, in English and German the colours between green and blue tend to be called teal or turquoise instead of blue and green, but the consensus across observers is not as high for these colour terms as for the eleven Basic Colour Terms (Lin, Luo, MacDonald, \& Tarrant, 2001; Lindsey \& Brown, 2014; Mylonas \& MacDonald, 2016; Witzel et al., 2008; Zimmer, 1982; Zollinger, 1984). Other examples are different terms for blue in Italian (Bimler \& Uusküla, 2016; Paggetti, Menegaz, \& Paramei, 2016; Sandford, 2012), in Japanese (Kuriki et al., 2017) and orange and brown in Chinese (Gao \& Sutrop, 2014). Using high individual consensus as a dichotomous, qualitative criterion risks excluding potentially important colour terms from investigations and this may misrepresent the colour vocabulary of a language.

\section{Problems with other criteria}

The Samoan language creates colour terms by duplicating other words (Snow, 1971). Such colour terms may not be considered as monolexemic, and yet they constitute proper colour terms in that they are specific for labelling colours (see discussion in Biggam, 2012, p.23).

Another problem may be the co-existence of two colour terms to denote a similar range of colours. Marron and brun in French are both used for brown, but for different kinds of objects (Forbes, 1979; for discussion see also Biggam, 2012, p.25). According to the criterion of hypernymy they cannot both be considered as basic colour terms. Yet, they both specifically describe colours and they are applied to many objects, hence fulfilling the criteria of context independence. Although "brun" is applied to a more limited range of objects than "marron", it is not completely clear which of these two terms should be the French basic colour term that corresponds to the English brown (Forbes, 1979). A similar case could 


\section{Misconceptions about colour categories}

be made for the co-existence of "lila" and "violett" in Swedisch (Taft \& Sivik, 1997) and German (Jones, 2013).

Still other issues are not clear. For example, based on their criteria Berlin \& Kay (1991 [1969], p.125) identified only six colour terms in Mandarin Chinese (黑 black, 白 white, 红 red, 绿 green, 黄 yellow, 藍 blue). However, Mandarin includes colour terms that correspond to each of the English basic colour terms ( 橙 orange, 灰 grey, 粉紅 pink, 紫 purple, 棕 brown; cf. Gao \& Sutrop, 2014). Like most Mandarin words, Mandarin colour terms are composed by two characters. Almost all of them include the character for colour (色), except for pink, which is composed by the characters for "light" and "red" (粉紅). So, these colour terms are not monolexemic in the strict sense. Nevertheless, all those colour terms are an integral part of the Mandarin colour vocabulary in the sense that they are commonly used by all Mandarin speakers and cannot be replaced with the basic colour terms suggested by Berlin \& Kay (1991 [1969], p.125).

\section{Summary and outlook}

In sum, the idea and definition of basic colour terms provided a first approach to identify the most important colour terms in a language. This made it possible to study colour categories systematically and to compare them across cultures. However, the criteria for basic colour terms should be considered as a heuristic, rather than a strict rule. It should be kept in mind that the concept of basicness may be influenced by the characteristics of the researchers' own language and may cover particularities of languages that are not captured by the conceptual criteria.

Furthermore, basicness is not simply a dichotomous, qualitative property of colour terms that defines whether a colour term is basic or not. Instead, basicness should be conceived as a continuous and gradual characteristic. Several promising quantitative approaches have been proposed to determine the degree of basicness of colour terms. These include simple measures of frequency, consistency and consensus in colour naming (Boynton \& Olson, 1990; Fider, Narens, Jameson, \& Komarova, 2017; Guest \& Van Laar, 2000; Mylonas \& MacDonald, 2016; Sturges \& Whitfield, 1997), clusters in multidimensional scaling of colour naming 


\section{Misconceptions about colour categories}

(Bimler \& Uusküla, 2016), the application of Zipf's law to colour naming frequencies (A. M. Brown et al., 2016; Kuriki et al., 2017; Lindsey \& Brown, 2014), and measures of colour term information (Gibson et al., 2017; Lindsey et al., 2015). Those quantitative measures may also circumvent some of the other problems with purely conceptual definitions of basic colour terms. In particular, they can reveal high levels of basicness for colour terms that would not be considered as basic according to the conceptual criteria of basic colour terms, for example because they are not monolexemic.

\section{Focal colours}

Besides categorical perception, focal colours have been another major approach to relate colour perception and categorisation. Much of the scientific and the broader literature present the term "focal colour" as a synonym for category prototype, be it by explicit definition or by tacit assumption (e.g. Glottopedia - Discovering Linguistics, 2013; Kay, 2015; Malkoc et al., 2005; Rosch Heider, 1971; Self, 2014). However, there is a fundamental difference. The notion of a category prototype is descriptive. The prototype of a colour category is the most typical colour, or best example, of that category, such as the green that is "greener" than all the other green shades in the green category. In contrast, the idea of focal colours conveys a particular theory about category prototypes (see also Abbott, Griffiths, \& Regier, 2016), even though this is not always explicit in the literature. Presenting the theoretical notion of focal colours as synonymous with the descriptive notion of category prototypes may give the impression that the theoretical ideas attached to focal colours are scientifically established facts. Yet, those ideas are still under investigation and the conceptual difference between focal colours and category prototypes remains important.

\section{The concept focal colours}

The concept of "focal colours" conveys the idea that category prototypes correspond to universal foci or focal points that are stable across languages, and that the typical colours have particular perceptual or cognitive properties according to which they have a special status among all colours. 


\section{Misconceptions about colour categories}

\section{Foci and focal points}

The idea of category foci in colour space had originally been introduced by Berlin and Kay (1991 [1969], p. 7) as a synonym of category prototypes. At the same time, the idea of foci and focal points in colour space was also used to describe the observation that the prototypes of colour terms across a sample of 20 languages seemed to cluster in colour space (Berlin and Kay, 1991 [1969], p. 13ff; Kay \& McDaniel, 1978; Rosch Heider, 1972b). As a result, the idea of "focality" got linked to the idea that prototypes across languages cluster around universal (i.e. cross-culturally stable) foci in colour space (Regier, Kay, \& Cook, 2005; (Regier et al., 2007). This idea has been supported by prototype choices of observers from 110 non-industrialised languages in the World Colour Survey (MacLaury, 1997; Regier et al., 2005). The prototype choices clustered around a subset of English category prototypes, namely those for red, yellow, and to a lesser extent for green and blue.

\section{Salience}

Elaborating on the idea of foci, the precise term "focal colour" was introduced (Rosch Heider, 1971, 1972b) to describe category prototypes as "salient areas of the color space which are universally the most linguistically "codable" and the most easily remembered." (cf. abstract of Rosch Heider, 1972b). While the original idea of focal colours concentrated on a cognitive kind of salience in language and memory, more recent proposals suggested that focal colours are also perceptually salient (Douven, 2017; Jameson \& D'Andrade, 1997; Regier et al., 2007). These studies observed that the red, yellow, green, and blue prototypes corresponded to local maxima in saturation ("bumps" in colour space; cf. Figure 3). For the sake of simplicity, saturation is used here interchangeably with chroma (for the precise difference see e.g. chapter 4 in Fairchild, 2013). Saturation is the amount of chromaticity of a colour, and corresponds to the difference of that colour from neutral (or achromatic) greyscale colours (Fairchild, 2013).

\section{Sensory singularity}

Another approach to focal colours, suggested that category prototypes of red, yellow, green, and blue had the perceptual property of being more stable and 


\section{Misconceptions about colour categories}

reliable across illumination changes. It has been proposed that surfaces that correspond to typical red, yellow, green, and blue have a high degree of sensory singularity, which means that they reflect light in a more predictable and reliable way (Philipona \& O'Regan, 2006; Vazquez-Corral, O'Regan, Vanrell, \& Finlayson, 2012). Studies on the constancy of colour categories across illuminations supported this idea since they suggested that categorisation is more stable for colours around category prototypes than for colours at the category boundaries (Olkkonen, Witzel, Hansen, \& Gegenfurtner, 2009; Olkkonen et al., 2010). If the perception of category prototypes was particularly stable and reliable across illumination changes, they could work as perceptual anchors for colour categories (Witzel et al., 2015; Witzel, Maule, \& Franklin, 2013).

\section{Criticisms and open questions}

At the same time, there is also evidence against the universality and the special status of category prototypes.

\section{Colour-opponency}

First of all, typical red, yellow, green, and blue do not correspond to the poles of the cone-opponent dimensions that result from the second-stage mechanisms of colour vision (cf. above Malkoc et al., 2005; Webster, Miyahara, Malkoc, \& Raker, 2000; Witzel \& Gegenfurtner, 2013; 2018).

Furthermore, it is not clear whether the prototypes of red, yellow, green, and blue correspond to unique hues and Hering colours. Unique hues and Hering colours are supposed to be the points of references of subjective colour appearance (Kuehni, 2014; Valberg, 2001). In English, the hues of the prototypes of red, yellow, green, and blue seem to coincide with unique hues (Kuehni, 2001; Miyahara, 2003). Differences have only been observed between typical and unique green (Kuehni, 2001). There are also many cultures that combine greenish and bluish colours in one category ("grue"), which implies that they do not have separate category prototypes that correspond to unique green and blue (Berlin \& Berlin, 1975; Bornstein, 1973; Gibson et al., 2017; Kay, 1975; Lindsey \& Brown, 2002; Lindsey et al., 2015). 


\section{Misconceptions about colour categories}

\section{Saturation}

Another issue concerns the impact of stimulus sampling on the salience of English category prototypes (for a thorough discussion see Witzel, in press). The studies on focal colours used the set of maximally saturated Munsell chips. This stimulus set features local peaks of saturation close to many of the English category prototypes (Collier, 1973; Jameson \& D'Andrade, 1997; Regier et al., 2007). The variation of saturation in that stimulus set is correlated with prototype choices in the World Colour Survey (Witzel et al., 2015). It is also correlated with the consistency of categorisation (Olkkonen et al., 2010; Roberson et al., 2005; Witzel, 2016, in press), which partly reflects the degree of membership and typicality of each colour. It had been found that prototype choices for a set of more uniformly saturated Munsell chips were roughly similar to those observed with maximally saturated Munsell chips (Collier et al., 1976). Nevertheless, differences in the variation of saturation influence which hues observers chose as category prototypes (Witzel \& Hammermeister, 2017). This latter observation suggests a causal effect of saturation on prototype choices that might explain the observed correlations between saturation and prototype choices.

One reason for the role of saturation in typicality judgments could be that observers choose saturated prototypes for chromatic categories in order to distinguish them from achromatic categories (i.e. black, grey, and white). Another explanation could be that saturation together with lightness determines the difference of a colour from an achromatic grey background. According to definitions of perceptual salience in vision research (Itti \& Koch, 2001), this contrast to the background would make colours perceptually salient. These salient colours "jump out to the eye" and this could make them more likely to be chosen in a colour selection task used to measure category prototypes. Hence, the role of chroma, saturation and perceptual salience could potentially explain the statistical regularities in categorisation across cultures and languages (see e.g. Berlin \& Kay, 1969; Kay \& Regier, 2003; Lindsey \& Brown, 2006, 2009; Lindsey et al., 2015). However, the local maxima of the maximally saturated Munsell chips do not represent a property of the human colour perception, but are a feature of the Munsell system (Witzel \& Franklin, 2014; Witzel, Maule, et al., 2013; Witzel, Maule, \& Franklin, under revision). Sensory singularities, i.e. the predictability of 


\section{Misconceptions about colour categories}

the colour signal across illumination changes, strongly depend on chroma, and the relationship between sensory singularities and category prototypes breaks down when controlling saturation (Witzel et al., 2015). Studies on colour constancy further undermine the idea that the perception of category prototypes is particularly stable across illumination changes (Weiss, Witzel, \& Gegenfurtner, 2017; Witzel, Van Alphen, Godau, \& O'Regan, 2016).

\section{Variation across languages}

The existence of category prototypes that do not correspond to focal red, yellow, green, and blue further undermines the idea that focal colours are universal points of reference for colour categorisation. In English, such non-focal prototypes are typical purple, pink, orange, and brown (plus grey as an achromatic category). In contrast to red, yellow, green, and blue (Douven, 2017; Regier et al., 2007), those other English prototypes cannot be predicted based on the within- and across category differences in the set of maximally saturated Munsell chips (Jraissati \& Douven, 2017). At the same time, there are languages in which English red, yellow, green, and blue are not the most salient colours. In those languages, observers showed superior recognition memory for colours that were most typical for their own colour categories (Roberson et al., 2005; Roberson et al., 2000). A recent approach provides an alternative to the idea that categories form around universal focal colours (Abbott et al., 2016). It suggests instead that category prototypes are determined as the most representative colours of categories in the sense that they are closest to members of their own, and furthest to members of other categories. This approach is better in modelling category prototypes than predictions based on saturation or on universal focal colours. Instead of explaining categories through prototypes, this approach predicts prototypes based on the given set of language-specific categories.

\section{Summary}

In sum, many sources conflate focal colours and category prototypes. However, the notion of focal colours comes with the assumptions that typical colours have particular properties due to which they are universal points of reference for colour categorisation. It is still under investigation whether category prototypes have the 


\section{Misconceptions about colour categories}

universal properties that would qualify them as focal colours. On the one hand, prototype choices across languages cluster around the prototypes of English red, yellow, green, and blue. On the other hand, it is not yet clear, whether categories form around those prototypes, whether those prototypes result from systematic patterns of categorisation, or whether those prototype clusters are simply due to the variation of saturation in the classical stimulus set used on almost all crosscultural studies on colour categorisation. To exclude the latter possibility, it seems advisable to use stimulus sets other than maximally saturated Munsell chips and to consider the potential role of saturation in colour categorisation when designing the stimulus sets and when analysing the resulting data. Until these issues are clarified it is wise to distinguish between the descriptive term "prototype" and the hypothetical idea of focal colours.

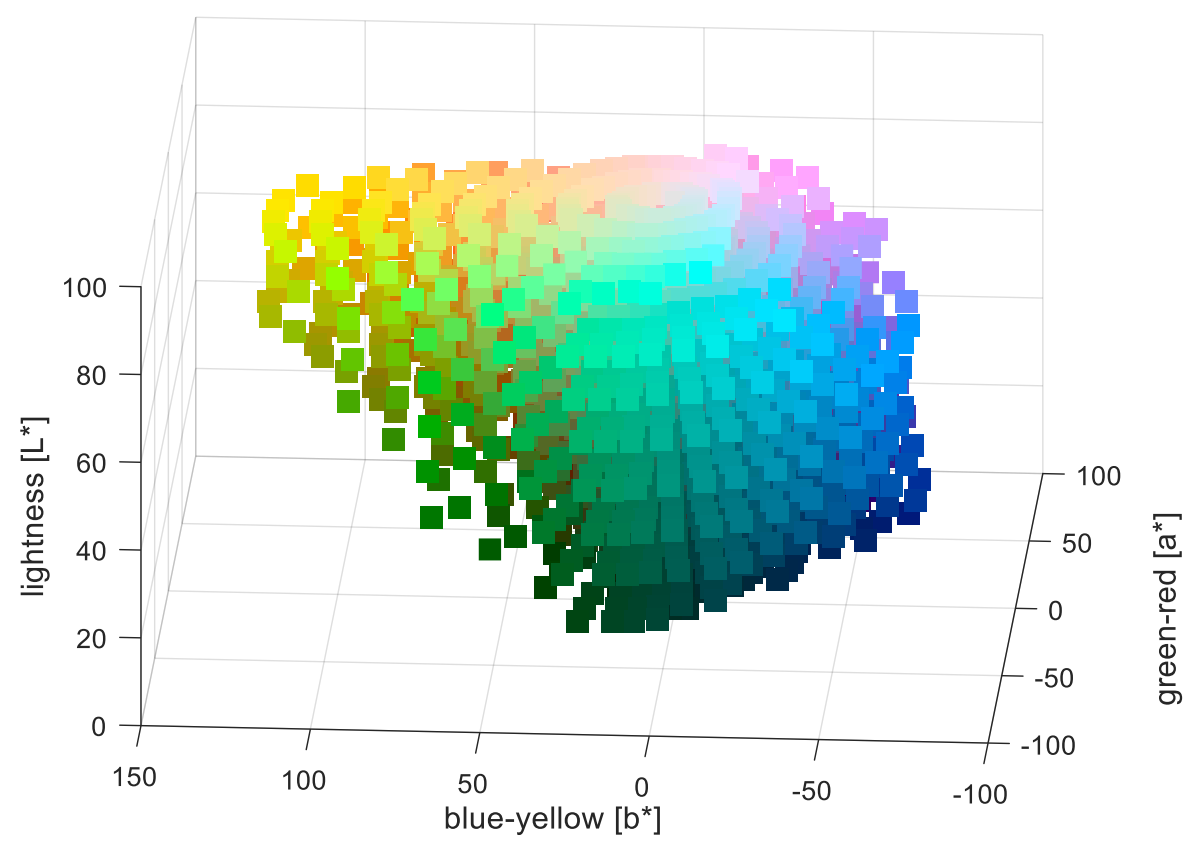

Figure 3. Bumps in the Munsell Colour Solid. Each Munsell chip is represented as a square in CIELAB space. All chips together they constitute the Munsell Colour Solid. The diagram is rotated to highlight the saturation bump for yellow (which is particularly protruding) and blue (which is the small bump on the right). Note that displayed colours can vary depending on print and display devices. Displayed colours are for approximate illustration only.

\section{Universalism vs. Relativism}

The origin of colour categories has been the most important research question on colour categorisation. Historically, research on the origin of colour categorisation revolved around a debate between universalists and linguistic relativists (e.g. 


\section{Misconceptions about colour categories}

Gumperz \& Levinson, 1991; Kay \& Kempton, 1984; Kay \& Regier, 2006; Regier $\&$ Kay, 2009). As mentioned in the introduction, the position of linguistic relativism holds that colour categories are determined by language, implying that colour categories vary across languages and that those categories shape how speakers of the respective language perceive and conceptualize the world (e.g. Gentner \& Goldin-Meadow, 2003; Gumperz \& Levinson, 1991; Wolff \& Holmes, 2011). The opposite position, universalism, holds that perception determines universal categories independent of language, and that language refers to these categories through labelling. Besides theories about focal colours (see section above), one of the most important universalist approaches was the idea that the evolution of colour categories in all languages follows a fixed sequence that started with only two basic colour terms and culminated in the eleven basic colour terms of English.

Still today, the idea that the universalism-relativism debate is at the core of colour category research is reported in many scientific publications and beyond (Davidoff, 1999; Deutscher, 2011; Gibson et al., 2017; Lindsey et al., 2015; Loreto et al., 2012). The idea that colour term evolution follows a fixed sequence from two to eleven colour terms has also been communicated to a broader scientific audience (e.g. Gage, 1999; E. B. Goldstein, 2014; Hansen, 2010; Hardin, 1988, 2014; Loreto et al., 2012) and to the non-scientific public (e.g. Deutscher, 2011; Schneider, 2002; Vox, 2016; Welsch \& Liebmann, 2004). From those sources, it might appear that the origin of colour categories can be understood by evaluating evidence for and against the universalist and the relativist view, respectively. Some of those sources also suggest that the eleven English basic colour terms constitute the maximum number of basic colour terms and the end point of the evolutionary sequence. However, current research on the origin of colour categories contradicts the fundamental logic behind the universalism-realism debate and led to important updates of the evolutionary sequence. 


\section{Misconceptions about colour categories \\ The logic of the universalism-realism debate}

\section{The origins of the debate}

Empirical research on colour categories began with the investigation of linguistic relativity and the Sapir-Whorf-Hypothesis (R. W. Brown \& Lenneberg, 1954; Lenneberg, 1953). Linguistic conventions were assumed to shape colour categories depending on the historical and cultural particularities of the respective language. Thus, the variation of colour categories across languages was taken as evidence for (linguistic) relativism.

When examining 98 languages, Berlin and Kay (1991 [1969]) observed that the basic colour terms of these languages corresponded to categories that were a systematic recombination of the categories of the eleven English Basic Colour Terms. Berlin and Kay (1991 [1969]) found one example, the Dungum Dani, with only two colour terms (cf. section on basic colour terms). These were considered the lowest level in an evolutionary sequence of colour category differentiation that culminates in the distinction of all eleven English colour categories. This evolutionary sequence was an elaboration of an evolutionary sequence of colour terms proposed in the 19th century by Lazarus Geiger (Deutscher, 2011; Saunders, 2000, 2007).

The evolutionary sequence implied two claims. On the one hand, the evolutionary stages aimed at capturing cross-cultural similarities in colour categorization. On the other hand, the sequential order of the evolutionary sequence described a process of colour term evolution that starts with few and develops towards many colour terms. These two claims contradicted the idea that colour categories are arbitrarily determined by linguistic convention, as claimed by linguistic relativism (e.g. Kay \& Kempton, 1984; Kay \& McDaniel, 1978; Rosch Heider, 1972b). A debate between universalists and (linguistic) relativists developed.

\section{The issues at stake}

The universalism-realism debate implies a polarized contrast between nature and nurture. Relativists claimed language, perception, and thought are shaped by history and culture. In contrast, the universalist position explained cross-cultural patterns of categorisation by biological, innate determinants, such as the 


\section{Misconceptions about colour categories}

characteristics of colour perception (e.g. Hardin, 2005; Kay, Berlin, \& Merrifield, 1991; Kay \& McDaniel, 1978). At the same time, the discussion about the evolutionary sequence involves claims about the process of colour term evolution. Thus, the universalism-realism debate combines the question of cross-cultural variation of colour categorization with claims about the determinants of colour categories, and the process of colour term evolution. However, empirical findings accumulated in the course of the universalism-relativism debate are complex and call for disentangling the different claims attached to the positions of the original debate (see also Jraissati, 2014).

\section{Nature vs. nurture}

\section{Cross-cultural variation}

By now, it has become clear that there are differences in colour categorization across languages and cultures (e.g. Jameson \& Alvarado, 2003; Roberson et al., 2005; Roberson et al., 2000). These differences are obvious from the fact that the number of colour categories and the boundaries between categories strongly vary across cultures (see already Berlin \& Kay, 1991 [1969]; Webster, \& Kay, 2007). At the same time, there is also ample evidence for statistical regularities in colour categorisation across fundamentally different cultures and languages (see e.g. Berlin \& Kay, 1991 [1969]; Kay \& Regier, 2003; Lindsey \& Brown, 2006, 2009; Lindsey et al., 2015; Regier et al., 2005). Statistical regularities imply that there is variation across languages, but that regularities appear when analyzing the variation across many languages.

The cross-cultural regularities may be captured by a few patterns or motifs. These motifs are two to four generic sets of colour categories that underlie the systematic variation of colour categorisation across individuals and across cultures (A. M. Brown et al., 2016; Gibson et al., 2017; Kuriki et al., 2017; Lindsey \& Brown, 2009, 2014; Lindsey et al., 2015). According to those motifs, black, white, and red are most likely to be distinguished even in languages that have few categories, and in many languages green and blue are combined in a composite "grue" category. It has also been argued that the full set of English categories is latent in emerging categories even if categorisation is not yet 


\section{Misconceptions about colour categories}

consistent across individuals (Gibson et al., 2017; Haynie \& Bowern, 2016; Lindsey \& Brown, 2009; Lindsey et al., 2015).

One caveat may be raised with respect to the colour stimuli: Almost all the studies that found cross-cultural regularities used the set of maximally saturated Munsell chips, implying that saturation, salience and distances between colour chips varied in a way that is specific to that stimulus sample. Given the role of saturation, salience and colour differences in perception and categorisation (see section on focal colours) it would be advisable to replicate the evidence for cross-cultural motifs with other stimulus sets, in which maximal saturation does not coincide with English basic colour terms (Witzel, 2016, in press; Witzel \& Hammermeister, 2017).

\section{Determinants of colour categories}

Cross-cultural regularities do not necessarily reflect innate, biological determinants (Kay, 2015; Lindsey et al., 2015; Roberson, 2005; Steels \& Belpaeme, 2005). They may also evolve as a result of ecological factors ("visual diet"), i.e. the distribution of colours in the environment (Roberson, 2005; Yendrikhovskij, 2001), and cultural factors, such as the cultural function of colours when communicating about coloured objects and materials (Baddeley \& Attewell, 2009; Gibson et al., 2017; Kirby, Dowman, \& Griffiths, 2007; Regier, Kemp, \& Kay, 2015; Witzel, 2012). Computer simulations have shown that crosscultural constraints may result from a dynamic interaction between biological, cultural and ecological constraints (Baronchelli et al., 2010; Kirby et al., 2007; Loreto et al., 2012; Steels \& Belpaeme, 2005). Still other computational simulations suggested that cross-cultural constrains may emerge out of the variation in colour perception across individuals, particularly in the presence of colour deficiencies in a population (Jameson \& Komarova, 2009a, 2009b). It is still an open question which determinants are at the origin of colour categories.

\section{Implications}

These observations do not only highlight that many questions still remain open; they also call for a fundamental change in perspective. Evidence for variation and 


\section{Misconceptions about colour categories}

statistical regularities across languages and cultures partially support the claims of both, the universalist and the relativist position. It has been accepted that colour categories are neither completely universal nor completely relative to culture and language (Abbott et al., 2016; Jraissati, 2014; Kay \& Regier, 2006; Kuehni, 2007; Regier \& Kay, 2009; Roberson, 2005). This observation contradicts a clear-cut dichotomy between universalism and relativism, and between nature and nurture, hence making a polarized discussion obsolete (Bornstein, 2007; Kay \& Regier, 2006; Regier \& Kay, 2009). To explain the origin of colour categories, it has been suggested that "nature proposes" certain universal constraints and "nurture disposes" of these constrains depending on culture-specific learning (Kay \& Regier, 2006).

However, the results on the determinants of colour categories do not just moderate the extreme positions of the universalist-relativist debate. They also dissociate evidence for cross-cultural variation from claims on the determinants of colour categorization, and showed that cross-cultural variation alone does not establish where colour categories come from. By doing so they undermine the fundamental assumption of the universalism-relativism debate according to which crosscultural variation is taken to reflect the linguistic or cultural origin of colour categories (R. W. Brown \& Lenneberg, 1954; Lenneberg, 1953). Thus, investigations on the origin of colour categories should clearly distinguish between patterns of cross-cultural variation and the determinants that are at the source of the variation.

\section{The evolutionary sequence}

Following the logic of the above paragraph, the idea of an evolutionary sequence does not necessarily imply claims about the determinants that shape the evolutionary sequence. Instead, it raises additional questions because it makes claims about evolutionary stages and the process of evolution. These claims rely on evidence for cross-cultural patterns; but cross-cultural patterns alone do not prove the existence and validity of an evolutionary sequence.

The original proposals for an evolutionary sequence have been strongly criticized throughout the universalism-realism debate (cf. critics of Basic colour terms above or Kay, 2015, p. 247-248). However, the evolutionary sequence has been 


\section{Misconceptions about colour categories}

progressively updated and refined in response to its critics and to accommodate new empirical evidence, mainly from the World Color Survey (for overview see Kay, 2015; Kay et al., 2011; Kay \& Maffi, 1999).

\section{Evolutionary stages}

The newest version of the evolutionary sequence consists of only five evolutionary stages and is limited to the development of colour terms for the six Hering colors, i.e. white, red, yellow, green, blue, and black (cf. Figure 11.5 and 11.6 in Kay, 2015; Kay et al., 2011, p.11ff). The first, second and last stage describe a single set of categories, including two categories (first stage), three (second) and six categories (last stage). The two intermediate stages propose three possible sets of categories each, leaving space for considerable cross-cultural variation.

Thus, the newest version of the evolutionary sequence does neither account for all possible sets of basic colour terms nor for the eleven English basic colour terms. The minimum and maximum number of basic colour terms per set remains unclear. There are languages that have more than eleven basic colour terms. In particular, several languages have twelve basic colour terms because they distinguish two kinds of blue, such as Russian (Corbett \& Morgan, 1988; Moss, Davies, Corbett, \& Laws, 1990; Paramei, 2005; Paramei, Griber, \& Mylonas, 2018), Greek (Androulaki et al., 2006; Athanasopoulos, 2009), and Turkish (Özgen \& Davies, 1998). It has been argued that there are fifteen Basic Colour Terms in Korean (Y. S. Kim, Pak, \& Lee, 2001; Pak, Kim, Kim, \& Lee, 2004; Roberson et al., 2008; Tyson, 1998; but for another opinion see A. I. Kim, 1985). These observations suggest that the eleven English basic colour terms do not constitute the maximum number of basic colour terms and the end point of colour term evolution. Concerning the minimum number of basic color terms, the Dugum Dani are the only known example of a language with only two colour terms (Kay, 2015). Thus, the idea that the Dani might have more than just two colour terms undermines the first stage of the evolutionary sequence (Groh, 2016). In any case, the specification of the number of basic colour terms also depends on the definition of basic colour terms, which is still under investigation (cf. section on basic colour terms). 


\section{Misconceptions about colour categories}

Furthermore, the idea of an evolutionary sequence implies a hierarchical order of the stages according to which some stages are "lower" and others "higher" in the evolutionary sequence. As support for the hierarchical ordering of colour terms, associations have been found in several languages between the frequency of usage of different colour terms and their order along the evolutionary sequence (e.g. McManus, 1997). Similarities to the evolutionary sequence have also been observed in the history of English (Casson, 1997), and a study found that observers follow that sequence when dividing Munsell chips in successively higher numbers of unlabelled categories (Boster, 1986). However, the ontogenetic development of colour term acquisition during childhood does not follow the evolutionary sequence (Pitchford \& Mullen, 2002; Witzel, Flack, \& Franklin, 2013). Although grey and brown tend to be learned later than other basic colour terms, the acquisition of the other basic colour terms does not follow a systematic order.

\section{Evolutionary process}

Apart from the evolutionary stages, another important update concerns the question of how new colour categories transition from one to another stage. The original idea was that colour categories of basic colour terms cover the whole perceptual colour space and that new colour categories evolve through partitioning, i.e. subdivisions of composite categories (partitioning hypothesis). Meanwhile, an alternative idea has been considered, the emergence hypothesis (Kay, 2015; Levinson, 2000). According to this idea, some languages do not have colour terms for all perceivable colours and new colour categories may emerge in regions of colour space that are not named or are named inconsistently (e.g. Everett, 2005; Hill, 2011; Kay, 2015; Levinson, 2000; Lindsey et al., 2015; Lindsey et al., 2016). For example, the analysis of latent categories in American English indicated that categories of new candidate basic colour terms (e.g. teal, peach) emerge at the boundary between categories (e.g. green and blue), not as a subdivision of existing categories (Lindsey \& Brown, 2014). At the same time, there was also evidence in that data for the partitioning hypothesis (Lindsey \& Brown, 2014). 


\section{Misconceptions about colour categories}

Furthermore, many linguistic theories suggest that languages develop from complex to more simple structures (e.g. Groh, 2016; Hardin \& Maffi, 1997). According to the evolutionary sequence, colour terms develop in the opposite direction, from a simple set of only a few categories towards a larger, more complex set of categories. The aforementioned latent patterns of colour terms with low consensus suggest that colour categories might evolve from simple composite to more diverse subdivided sets of categories (Gibson et al., 2017; Kuriki et al., 2017; Lindsey \& Brown, 2014; Lindsey et al., 2015; Lindsey et al., 2016). However, these latent patterns do not prove that category evolution necessarily follows a unidirectional process from simple to complex. A recent study (Haynie $\&$ Bowern, 2016) suggested that colour categories can develop in both directions. Based on a statistical model of colour term evolution in a particular language family (the Pama-Nyugan languages of Australia), it observed that colour terms can be lost during cultural evolution, implying that diverse category sets can develop into simpler sets with fewer categories. However, this idea has been questioned (Nash, 2017), and the directionality of colour term evolution remains open to further research.

\section{Summary}

In sum, the universalist-relativist debate assumes a contrast between Nature and Nurture implying a direct relationship between cross-cultural patterns and the determinants of colour categories. In addition, this debate is strongly associated with the idea of an evolutionary sequence, which was at the origin of the universalist approach to colour categorisation. As a result, the logic behind the universalism-realism debate ties together three different issues: the cross-cultural variation of colour categories, the evolutionary process, and the determinants that shape colour categorisation.

However, state-of-the-art research has shown that each of the above three issues is complicated and deserves proper scientific evaluation. First, while the crosscultural variation of colour categorisation is obvious, recent studies using advanced computational approaches identified cross- and intra-cultural patterns, or motifs. Second, the evolutionary sequence has been adapted to take recent evidence on cross-cultural patterns into account; but evidence for the evolutionary 


\section{Misconceptions about colour categories}

sequence itself is contradictory and incomplete. Even the newest version cannot account for the full diversity of colour categories across languages. Third, neither cross-cultural motifs nor evolutionary sequence allow for specifying the determinants at the origin of the colour categories. The precise role of biological, ecological, and cultural determinants in the evolution and formation of those motifs is yet under investigation.

Consequently, the origin of colour categorisation cannot be understood through the simple contrast between nature and nurture, implying that the debate between universalism and relativism is obsolete. State-of-the-art investigations on the origin of colour categories should overcome the universalism-realism dichotomy and consider the possibility that observed patterns may result from the complex interaction of multiple constraints and determinants.

\section{Conclusions}

This review provided an update on four of the most fundamental concepts about colour categorization that have led to misconceptions in the wider dissemination of research on colour naming and categorisation. First, considering colour as a prime example of categorical perception, communications to the broader public, scientific textbooks and many specialized articles on colour categorization describe colour perception as inherently categorical. Methodological shortcomings, conceptual misunderstandings and circularities in argumentation led to this deceptive conclusion. In fact, there is strong evidence that colour perception per se is not categorical, but effects of categories occur at a higher, more cognitive level, probably due to attention. Recent influential literature claimed that category effects are lateralized, but such lateralization effects are difficult to reproduce with highly controlled stimuli.

Second, the definition of basic colour terms allowed for comparing colour categories across languages. While this may be appropriate as a first approach to the cross-cultural comparison of colour naming, the uncritical application of the original criteria for basic colour terms has led to fundamental misrepresentations of languages, including the persistent myth that there are languages with only two basic colour terms, and that the maximum number of basic colour terms is eleven. 


\section{Misconceptions about colour categories}

More pertinent and culture-fair approaches are now available that allow for a rigorous empirical assessment and a graded concept of basic colour terms. Third, seminal studies observed regularities in colour categorization across languages and cultures. A particularly persistent idea claims that the most typical colours of colour categories are perceptually salient and should be considered as focal colours. However, one source for perceptual salience of category prototypes is due to the particular stimulus sample, a subset of the Munsell system, that has been used since the beginnings of empirical research on colour naming and that completely neglects the role of saturation. In fact, cross-cultural regularities may be partly explained by the variation of saturation in that classical stimulus set. The variation of saturation in that stimulus set, however, is not a feature of colour perception and categorization, but a peculiarity of that stimulus set and hence a methodological artefact that might have led to spurious cross-cultural regularities in colour naming.

Fourth, the universalist-relativist debate is over - not because it is solved, but because it is obsolete and unfit to unravel the origin of colour categories. The universalist-relativist debate confuses claims about cross-cultural variation, evolutionary processes and the determinants of colour categories. These three claims need to be disentangled because evidence for each of the claims is complicated and requires careful examination on its own. First, research has revealed both, language-specific variation and cross-cultural statistical regularities. Second, findings on the ontogenetic and phylogenetic development of languages and cross-cultural comparisons contradict the classical evolutionary model, according to which colour term evolution follows a fixed seven-stage sequence that culminates in the English basic colour terms. Instead, the reconceptualization of basic colour terms has led to new approaches to the understanding of colour term evolution. Third, several candidate ecological, cultural and biological determinants have been identified, but it is still under investigation which determinants actually explain the origin of colour categories. These updates on colour categories may be transferred to other domains of categorisation. Controlling stimulus differences in a perceptually meaningful way is important to all research on categorisation beyond colour. As with colour categories, it has been found that evidence for categorical perception of phonemes 


\section{Misconceptions about colour categories}

depends on the tasks used to measure category effects on perception (Gerrits \& Schouten, 2004; Schouten, Gerrits, \& van Hessen, 2003). It may be useful to target particular levels of perceptual processing of phoneme discrimination by choosing appropriate measures of perceptual reference and category-probing tasks. Measuring discrimination thresholds to control sensitivity and to test for high-level categorical facilitation should in principle be possible for a large range of perceptual domains. For example, this approach could be used to characterise category effects in phoneme perception (e.g. Liberman, Harris, Hoffman, \& Griffith, 1957), orientation perception (e.g. Franklin, Catherwood, Alvarez, \& Axelsson, 2010), haptic shape perception (e.g. Gaissert et al., 2012), face perception (e.g. Kikutani, Roberson, \& Hanley, 2008), the perception of animals (e.g. Gilbert, Regier, Kay, \& Ivry, 2008) and animal patterns (e.g. J. Goldstein \& Davidoff, 2008), and of novel stimuli (e.g. Holmes \& Wolff, 2012). Using these approaches to stimulus control allows us to assess whether or not the determinants of category effects are similar for different perceptual domains.

Furthermore, the development of empirical criteria for basic colour terms may be helpful for assessing basic terms and categories in a culture-fair way in other domains of perception, such as taste (e.g. Erickson, 2008) and smell (e.g. Majid \& Burenhult, 2014). For example, the criterion of inter-individual consensus has been used to compare the vocabulary between colour and smell across languages (Majid \& Burenhult, 2014). A standard set of empirical criteria could be designed that makes basic vocabularies across many different perceptual domains and modalities comparable. Such a set of criteria would open up new paths to compare linguistic descriptions for different modalities and to investigate the linguistic basis of cross-modal correspondences (e.g. Bremner et al., 2013; Deroy \& Spence, 2016).

\section{Acknowledgements}

I am grateful to Paul Kay, Delwin Lindsey, and Simon Cropper for helpful comments on the manuscript, and to J. Kevin O'Regan for suggesting the title of this paper. This work was supported by DFG Sonderforschungsbereich SFB TRR 135. 


\section{Misconceptions about colour categories}

\section{References}

Abbott, J. T., Griffiths, T. L., \& Regier, T. (2016). Focal colors across languages are representative members of color categories. Proceedings of the National Academy of Sciences, 113(40), 1117811183. doi:10.1073/pnas.1513298113

Alexander, M., \& Kay, C. (2014). The Spread of RED in the Historical Thesaurus of English. In W. Anderson, C. P. Biggam, C. Hough, \& C. Kay (Eds.), Colour Studies: A Broad Spectrum (pp. 126-139). Amsterdam: John Benjamins.

Alvarez, J., Clifford, A., Holmes, A., \& Franklin, A. (2012). Attention modulates hemispheric lateralisation of categorical colour search: An alternative account for 'Lateralised Whorf'. Paper presented at the Progress in Colour Studies 2012 (PICS12), Glasgow.

Androulaki, A., Gômez-Pestaña, N., Mitsakis, C., Jover, J. L., Coventry, K., \& Davies, I. R. L. (2006). Basic colour terms in Modern Greek - Twelve terms including two blues. Journal of Greek Linguistics, 7, 3-47.

Athanasopoulos, P. (2009). Cognitive representation of colour in bilinguals: The case of Greek blues. Bilingualism: Language and Cognition, 12, 83-95.

Auvray, M., Hanneton, S., Lenay, C., \& O'Regan, J. K. (2005). There is something out there: distal attribution in sensory substitution, twenty years later. J Integr Neurosci, 4(4), 505-521.

Bachy, R., Dias, J., Alleysson, D., \& Bonnardel, V. (2012). Hue discrimination, unique hues and naming. Journal of the Optical Society of America. A, Optics, image science, and vision, 29(2), A60-68. doi:10.1364/JOSAA.29.000A60

Baddeley, R., \& Attewell, D. (2009). The relationship between language and the environment: information theory shows why we have only three lightness terms. Psychological Science, 20(9), 1100-1107. doi:PSCI2412 [pii], 10.1111/j.1467-9280.2009.02412.x

Bae, G. Y., Olkkonen, M., Allred, S. R., \& Flombaum, J. I. (2015). Why some colors appear more memorable than others: A model combining categories and particulars in color working memory. Journal of Experimental Psychology: General, 144(4), 744-763. doi:10.1037/xge0000076 Baronchelli, A., Gong, T., Puglisi, A., \& Loreto, V. (2010). Modeling the emergence of universality in color naming patterns. Proceedings of the National Academy of Sciences USA, 107(6), 2403-2407. doi:10.1073/pnas.0908533107

Beare, A. C. (1963). Color-Name as a Function of Wave-Length. The American Journal of Psychology, 76(2), 248-256.

Berlin, B., \& Berlin, E. A. (1975). Aguaruna Color Categories. American Ethnologist, 2, 61-87.

Berlin, B., \& Kay, P. (1991 [1969]). Basic color terms: their universality and evolution. Berkeley, CA: University of California Press.

Biggam, C. P. (2012). The Semantics of Colour: A Historical Approach. Cambridge: Cambridge University Press.

Bimler, D. (2009). Linguistic and Perceptual Categories in Colour Vision: A Critical Review. Journal of Cognitive Science, 10(Special Issue: Color in Thought and Language). 


\section{Misconceptions about colour categories}

Bimler, D., \& Uusküla, M. (2016). A similarity-based cross-language comparison of basicness and demarcation of "blue" terms. Color Research \& Application, n/a-n/a. doi:10.1002/col.22076

Bornstein, M. H. (1973). Color vision and color naming: a psychophysiological hypothesis of cultural difference. Psychological Bulletin, 80(4), 257-285.

Bornstein, M. H. (1987). Perceptual categories in vision and audition Categorical perception: The groundwork of cognition. (pp. 287-300). New York, NY, US: Cambridge University Press.

Bornstein, M. H. (2007). Hue Categorization and Color Naming: Cognition to Language to Culture. In R. E. MacLaury, G. V. Paramei, \& D. Dedrick (Eds.), Anthropology of Color (pp. 327). Philadelphia: John Benjamins Publishing Company.

Bornstein, M. H., \& Korda, N. O. (1984). Discrimination and matching within and between hues measured by reaction times: some implications for categorical perception and levels of information processing. Psychological Research, 46(3), 207-222.

Bornstein, M. H., Kessen, W., \& Weiskopf, S. (1976). The categories of hue in infancy. Science, 191(4223), 201-202.

Boster, J. (1986). Can Individuals Recapitulate the Evolutionary Development of Color Lexicons? Ethnology, 25(1), 61-74.

Boynton, R. M., \& Kambe, N. (1980). Chromatic Difference Steps of Moderate Size Measured along Theoretically Critical Axes. Color Research \& Application, 5(1), 13-23.

Boynton, R. M., \& Olson, C. X. (1990). Salience of chromatic basic color terms confirmed by three measures. Vision Research, 30(9), 1311-1317. doi:0042-6989(90)90005-6 [pii]

Brainard, D. H. (2015). Color and the Cone Mosaic. Annual Review of Vision Science, 1(1), 519546. doi:doi:10.1146/annurev-vision-082114-035341

Bremner, A. J., Caparos, S., Davidoff, J., de Fockert, J., Linnell, K. J., \& Spence, C. (2013). "Bouba" and "Kiki" in Namibia? A remote culture make similar shape-sound matches, but different shape-taste matches to Westerners. Cognition, 126(2), 165-172.

doi:http://doi.org/10.1016/j.cognition.2012.09.007

Brown, A. M., Isse, A., \& Lindsey, D. T. (2016). The color lexicon of the Somali language. Journal of Vision, 16(5), 14-14. doi:10.1167/16.5.14

Brown, A. M., \& Lindsey, D. T. (2013). Infant color vision and color preferences: a tribute to Davida Teller. Visual Neuroscience, 30(5-6), 243-250. doi:10.1017/S0952523813000114

Brown, A. M., Lindsey, D. T., \& Guckes, K. M. (2011). Color names, color categories, and colorcued visual search: Sometimes, color perception is not categorical. Journal of Vision, 11(12).

Brown, R. W., \& Lenneberg, E. H. (1954). A study in language and cognition. Journal of Abnormal and Social Psychology, 49(3), 454-462.

Casson, R. W. (1997). Color shift: evolution of English color terms from brightness to hue. In C.

L. Hardin \& L. Maffi (Eds.), Color Categories in Thought and Language (pp. 224-239).

Cambridge: Cambridge University Press.

Cibelli, E., Xu, Y., Austerweil, J. L., Griffiths, T. L., \& Regier, T. (2016). The Sapir-Whorf Hypothesis and Probabilistic Inference: Evidence from the Domain of Color. PLoS One, 11(7), e0158725. doi:10.1371/journal.pone.0158725 


\section{Misconceptions about colour categories}

Collier, G. A. (1973). Review of "Basic Color Terms: Their Universality and Evolution". Language, 49(1), 245-248.

Collier, G. A., Dorflinger, G. K., Gulick, T. A., Johnson, D. L., McCorkle, C., Meyer, M. A., . . Yip, L. (1976). Further Evidence for Universal Color Categories. Language, 52(4), 884-890. Collins, J. A., \& Olson, I. R. (2014). Knowledge is power: how conceptual knowledge transforms visual cognition. Psychonomic Bulletin \& Review, 21(4), 843-860. doi:10.3758/s13423-013-05643

Conklin, H. C. (1973). Color Categorization. American Anthropologist, 75(4), 931-942.

Constable, M. D., \& Becker, S. I. (2017). Right away: A late, right-lateralized category effect complements an early, left-lateralized category effect in visual search. Psychonomic Bulletin \& Review, 1-9. doi:10.3758/s13423-017-1246-3

Corbett, G. G., \& Morgan, G. (1988). Colour terms in Russian: Reflections of typological constraints on a single language. Journal of Linguistics, 24, 31-64.

Crawford, T. D. (1982). Defining "basic color term". Anthropological Linguistics, 24, 338-343. Cropper, S. J., Kvansakul, J. G., \& Little, D. R. (2013). The categorisation of non-categorical colours: a novel paradigm in colour perception. PLoS One, 8(3), e59945. doi:10.1371/journal.pone.0059945

Cropper, S. J., \& Wuerger, S. M. (2005). The Perception of Motion in Chromatic Stimuli. Behavioral and Cognitive Neuroscience Reviews, 4(3), 192-217. doi:10.1177/1534582305285120 Davidoff, J., Davies, I. R. L., \& Roberson, D. (1999). Colour categories in a stone-age tribe. Nature, 398(6724), 203-204. doi:10.1038/18335

Davidoff, J., Goldstein, J., Tharp, I., Wakui, E., \& Fagot, J. (2012). Perceptual and categorical judgements of colour similarity. Journal of Cognitive Psychology, 24(7), 871-892. doi:10.1080/20445911.2012.706603 de Saussure, F. (1972). Cours de linguistique générale. Paris: Payot.

Dedrick, D. (1998). The Foundations of the Universalist Tradition in Color-Naming Research (and Their Supposed Refutation). Philosophy of the Social Sciences, 28(2), 179-204.

Deroy, O., \& Spence, C. (2016). Crossmodal Correspondences: Four Challenges. Multisens Res, 29(1-3), 29-48. doi:doi:https://doi.org/10.1163/22134808-00002488

Derrington, A. M., Krauskopf, J., \& Lennie, P. (1984). Chromatic mechanisms in the lateral geniculate nucleus of macaque. Journal of Physiology, 357, 241-265.

Deutscher, G. (2011). Through the Language Glass: Why the World Looks Different in Other Languages. London: Random House UK.

Douven, I. (2017). Clustering colors. Cognitive Systems Research, 45(Supplement C), 70-81. doi:https://doi.org/10.1016/j.cogsys.2017.05.004

Drivonikou, G. V., Kay, P., Regier, T., Ivry, R. B., Gilbert, A. L., Franklin, A., \& Davies, I. R. L. (2007). Further evidence that Whorfian effects are stronger in the right visual field than the left. Proceedings of the National Academy of Sciences USA, 104(3), 1097-1102. doi:0610132104 [pii] 10.1073/pnas.0610132104

Durbin, M. (1972). Review of Basic Color Terms. Semiotica, 6, 257-278. 


\section{Misconceptions about colour categories}

Eco, U. (1988). Le Signe: Histoire et Analyse d'un concept. Bruxelles: Labor.

Emery, K. J., Volbrecht, V. J., Peterzell, D. H., \& Webster, M. A. (2017). Variations in normal color vision. VII. Relationships between color naming and hue scaling. Vision Research. doi:https://doi.org/10.1016/j.visres.2016.12.007

Erickson, R. P. (2008). A study of the science of taste: On the origins and influence of the core ideas. Behavioral and Brain Sciences, 31(1), 59-75. doi:10.1017/S0140525X08003348

Eskew, R. T., Jr. (2009). Higher order color mechanisms: a critical review. Vision Research, 49(22), 2686-2704. doi:S0042-6989(09)00328-9 [pii] 10.1016/j.visres.2009.07.005

Everett, D. L. (2005). Cultural Constraints on Grammar and Cognition in Piraha. Current Anthropology, 46(4), 621-645.

Fairchild, M. D. (2013). Colour appearance models. Hoboken, Nj: Wiley.

Fider, N., Narens, L., Jameson, K. A., \& Komarova, N. L. (2017). Quantitative approach for defining basic color terms and color category best exemplars. Journal of the Optical Society of America A, 34(8), 1285-1300. doi:10.1364/JOSAA.34.001285

Firestone, C., \& Scholl, B. J. (2016). Cognition does not affect perception: Evaluating the evidence for "top-down" effects. Behavioral and Brain Sciences, 39, 1-77. doi:10.1017/S0140525X15000965

Forbes, I. (1979). The Terms "Brun" and "Marron" in Modern Standard French. Journal of Linguistics, 15(2), 295-305.

Foster, D. H. (2011). Color constancy. Vision Research. doi:S0042-6989(10)00440-2 [pii] 10.1016/j.visres.2010.09.006

Franklin, A. (2009). Pre-linguistic categorical perception of colour cannot be explained by colour preference: Response to Roberson and Hanley. Trends in Cognitive Sciences, 13(12), 501-502. doi:10.1016/j.tics.2009.10.006

Franklin, A., Catherwood, D., Alvarez, J., \& Axelsson, E. (2010). Hemispheric asymmetries in categorical perception of orientation in infants and adults. Neuropsychologia, 48(9), 2648-2657. doi:S0028-3932(10)00192-2 [pii], 10.1016/j.neuropsychologia.2010.05.011

Franklin, A., Clifford, A., Williamson, E., \& Davies, I. R. L. (2005). Color term knowledge does not affect categorical perception of color in toddlers. Journal of Experimental Child Psychology, 90(2), 114-141. doi:S0022-0965(04)00147-X [pii] 10.1016/j.jecp.2004.10.001

Franklin, A., \& Davies, I. R. L. (2004). New evidence for infant colour categories. British Journal of Developmental Psychology, 22, 349-377.

Franklin, A., Drivonikou, G. V., Bevis, L., Davies, I. R. L., Kay, P., \& Regier, T. (2008).

Categorical perception of color is lateralized to the right hemisphere in infants, but to the left hemisphere in adults. Proceedings of the National Academy of Sciences USA, 105(9), 3221-3225. doi:0712286105 [pii] 10.1073/pnas.0712286105

Franklin, A., Drivonikou, G. V., Clifford, A., Kay, P., Regier, T., \& Davies, I. R. L. (2008). Lateralization of categorical perception of color changes with color term acquisition. Proceedings of the National Academy of Sciences USA, 105(47), 18221-18225. doi:0809952105 [pii] 10.1073/pnas.0809952105 


\section{Misconceptions about colour categories}

Franklin, A., Pilling, M., \& Davies, I. R. L. (2005). The nature of infant color categorization: evidence from eye movements on a target detection task. Journal of Experimental Child Psychology, 91(3), 227-248. doi:S0022-0965(05)00053-6 [pii] 10.1016/j.jecp.2005.03.003 Franklin, A., Wright, O., \& Davies, I. R. L. (2009). What can we learn from toddlers about categorical perception of color? Comments on Goldstein, Davidoff, and Roberson. Journal of Experimental Child Psychology, 102(2), 239-245. doi:S0022-0965(08)00120-3 [pii] 10.1016/j.jecp.2008.08.003

Gage, J. (1999). Color and Culture - Practice and Meaning from Antiquity to Abstraction. Berkeley, CA: University of California Press.

Gaissert, N., Waterkamp, S., Fleming, R. W., \& Bulthoff, I. (2012). Haptic categorical perception of shape. PLoS One, 7(8), e43062. doi:10.1371/journal.pone.0043062

Gao, J., \& Sutrop, U. (2014). The basic color terms of Mandarin Chinese: A theory-driven experimental study. Studies in Language, 38(2), 335-359. doi:10.1075/s1.38.2.03gao

Gegenfurtner, K. R., \& Kiper, D. C. (2003). Color Vision. Annual Review of Neuroscience, 26(1), 181-206.

Gentner, D., \& Goldin-Meadow, S. (2003). Whiter Whorf. In D. Gentner \& S. Goldin-Meadow (Eds.), Language in Mind: Advances in the study of language and thought (pp. 3-14). Cambridge, MA: MIT Press.

Gerhardstein, P., Renner, P., \& Rovee-Collier, C. (1999). The roles of perceptual and categorical similarity in colour pop-out in infants. British Journal of Developmental Psychology, 17(3), 403420. doi:10.1348/026151099165366

Gerrits, E., \& Schouten, M. E. (2004). Categorical perception depends on the discrimination task. Perception and Psychophysics, 66(3), 363-376.

Gibson, E., Futrell, R., Jara-Ettinger, J., Mahowald, K., Bergen, L., Ratnasingam, S., . . Conway, B. R. (2017). Color naming across languages reflects color use. Proceedings of the National Academy of Sciences. doi:10.1073/pnas.1619666114

Giesel, M., Hansen, T., \& Gegenfurtner, K. R. (2009). The discrimination of chromatic textures. Journal of Vision, 9(9), 11 11-28. doi:10.1167/9.9.11/9/9/11/ [pii]

Gilbert, A. L., Regier, T., Kay, P., \& Ivry, R. B. (2006). Whorf hypothesis is supported in the right visual field but not in the left. Proceedings of the National Academy of Sciences USA, 103(2), 489-494. doi:0509868103 [pii] 10.1073/pnas.0509868103

Gilbert, A. L., Regier, T., Kay, P., \& Ivry, R. B. (2008). Support for lateralization of the Whorf effect beyond the realm of color discrimination. Brain Language, 105(2), 91-98. doi:S0093934X(07)00108-3 [pii], 10.1016/j.bandl.2007.06.001

Gilberthorpe, E. (2017). New Guinea's indigenous tribes are alive and well (just don't call them 'ancient'). The Conversation. Retrieved from https://theconversation.com/new-guineasindigenous-tribes-are-alive-and-well-just-dont-call-them-ancient-75888 Glottopedia - Discovering Linguistics. ( 2013). Focal colors. Retrieved from http://www.glottopedia.org/index.php/Focal_Colors 


\section{Misconceptions about colour categories}

Goldstein, E. B. (2014). Sensation and Perception (9 ed.). Belmore, CA: Wadsworth, Cengage Learning.

Goldstein, J., \& Davidoff, J. (2008). Categorical perception of animal patterns. British Journal of Psychology, 99(Pt 2), 229-243. doi:10.1348/000712607X228555

Goldstone, R. L. (1994a). The role of similarity in categorization: providing a groundwork. Cognition, 52(2), 125-157. doi:https://doi.org/10.1016/0010-0277(94)90065-5

Goldstone, R. L. (1994b). Influences of categorization on perceptual discrimination. Journal of Experimental Psychology: General, 123(2), 178-200.

Goldstone, R. L. (1995). Effects of Categorization on Color Perception. Psychological Science, 6(5), 298-304.

Goldstone, R. L., \& Hendrickson, A. T. (2010). Categorical perception. Wiley Interdisciplinary Reviews: Cognitive Science, 1(1), 69-78. doi:10.1002/wcs.26

Grandison, A., Sowden, P. T., Drivonikou, V. G., Notman, L. A., Alexander, I., \& Davies, I. R. (2016). Chromatic Perceptual Learning but No Category Effects without Linguistic Input.

Frontiers in Psychology, 7, 731. doi:10.3389/fpsyg.2016.00731

Groh, A. (2016). Culture, Language and Thought: Field Studies on Colour Concepts. Journal of Cognition and Culture, 16(1-2), 83-106. doi:doi:http://dx.doi.org/10.1163/15685373-12342169 Guest, S., \& Van Laar, D. (2000). The structure of colour naming space. Vision Research, 40(7), 723-734. doi:S0042-6989(99)00221-7 [pii]

Gumperz, J. J., \& Levinson, S. C. (1991). Rethinking Linguistic Relativity. Current Anthropology, 32(5), 613-623. doi:10.1086/204009

Hansen, T. (2010). Color naming. In E. B. Goldstein (Ed.), SAGE Encyclopedia of Perception (pp. 265-266). Thousand Oaks, CA: SAGE.

Hansen, T., \& Gegenfurtner, K. R. (2013). Higher order color mechanisms: evidence from noisemasking experiments in cone contrast space. Journal of Vision, 13(1). doi:10.1167/13.1.26

Hardin, C. L. (1988). Color for Philosophers: Unweaving the Rainbow. Indianapolis, ID: Hackett Publishing Company.

Hardin, C. L. (2005). Explaining Basic Color Categories. Cross-Cultural Research, 39(1), 72-87.

Hardin, C. L. (2014). Berlin and Kay Theory. In R. Luo (Ed.), Encyclopedia of Color Science and Technology (pp. 1-4). New York, NY: Springer New York.

Hardin, C. L., \& Maffi, L. (Eds.). (1997). Color categories in thought and language. Cambridge, UK: Cambridge University Press.

Harnad, S. (1987). Psychophysical and cognitive aspects of categorical perception: A critical overview. In S. Harnad (Ed.), Categorical Perception: The Groundwork of Cognition (pp. 287301). New York: Cambridge University Press.

Harnad, S. (2017). Chapter 2 - To Cognize is to Categorize: Cognition is Categorization A2 Cohen, Henri. In C. Lefebvre (Ed.), Handbook of Categorization in Cognitive Science (Second Edition) (pp. 21-54). San Diego: Elsevier.

Harris, J. (2014). Sensation and perception. London: Sage. 


\section{Misconceptions about colour categories}

Hartcher-O'Brien, J., \& Auvray, M. (2014). The Process of Distal Attribution Illuminated Through Studies of Sensory Substitution. Multisens Res, 27(5-6), 421-441.

doi:doi:http://dx.doi.org/10.1163/22134808-00002456

Haynie, H. J., \& Bowern, C. (2016). Phylogenetic approach to the evolution of color term systems. Proceedings of the National Academy of Sciences, 113(48), 13666-13671.

doi:10.1073/pnas.1613666113

Heider, K. G. (1971). The Dugum Dani: A Papuan culture in the highlands of west new Guinea. Chicago: Aldine.

Hickerson, N. P. (1971). Review of Basic Color Terms. International Journal of American Linguistics, 37, 257-270.

Hickerson, N. P. (1980). Review of "Voir et nommer les couleurs". Language in Society, 9(2), 255-267.

Hill, C. (2011). Named and Unnamed Spaces: Color, Kin, and the Environment in Umpila. The Senses and Society, 6(1), 57-67. doi:10.2752/174589311X12893982233759

Holmes, K. J., Moty, K., \& Regier, T. (2017). Revisiting the role of language in spatial cognition: Categorical perception of spatial relations in English and Korean speakers. Psychonomic Bulletin \& Review, 1-6. doi:10.3758/s13423-017-1268-x

Holmes, K. J., \& Wolff, P. (2012). Does Categorical Perception in the Left Hemisphere Depend on Language? Journal of Experimental Psychology: General. doi:10.1037/a0027289

Holtsmark, T., \& Valberg, A. (1969). Colour discrimination and hue. Nature, 224(5217), 366-367. Hunt, R. W. G., \& Pointer, M. R. (2011). Measuring Colour (4 ed.). Chichester, UK: John Wiley \& Sons.

Itti, L., \& Koch, C. (2001). Computational modelling of visual attention. Nature Reviews Neuroscience, 2(3), 194-203.

Jameson, K. A., \& Alvarado, N. (2003). Differences in color naming and color salience in Vietnamese and English. Color Research \& Application, 28, 113-138.

Jameson, K. A., \& D'Andrade, R. G. (1997). It's not really red, green, yellow, blue: an inquiry into perceptual color space. In C. N. Hardin \& L. Maffi (Eds.), Color categories in thought an language (pp. 295-319). Cambridge, UK: Cambridge University Press.

Jameson, K. A., \& Komarova, N. L. (2009a). Evolutionary models of color categorization. I. Population categorization systems based on normal and dichromat observers. Journal of the Optical Society of America A, 26(6), 1414-1423. doi:180032 [pii]

Jameson, K. A., \& Komarova, N. L. (2009b). Evolutionary models of color categorization. II.

Realistic observer models and population heterogeneity. Journal of the Optical Society of America A, 26(6), 1424-1436. doi:180033 [pii]

Jones, W. J. (2013). German colour terms : a study in their historical evolution from earliest times to the present (Vol. 119). Amsterdam u.a.

Jraissati, Y. (2014). Proving universalism wrong does not prove relativism right: Considerations on the ongoing color categorization debate. Philosophical Psychology, 27(3), 401-424.

doi:10.1080/09515089.2012.733815 


\section{Misconceptions about colour categories}

Jraissati, Y., \& Douven, I. (2017). Does optimal partitioning of color space account for universal color categorization? PLoS One, 12(6), e0178083. doi:10.1371/journal.pone.0178083

Kay, P. (1975). Synchronic variability and diachronic change in color terms. Language in Society, 4, 257-270.

Kay, P. (2015). Universality of color categorization. In A. J. Elliot, A. Franklin, \& M. D. Fairchild (Eds.), Handbook of Color Psychology (pp. 243-244). Cambridge: Cambridge University Press.

Kay, P., Berlin, B., \& Merrifield, W. (1991). Biocultural implications of systems in color naming. Journal of Linguistic Anthropology, 1(1), 12-25.

Kay, P., Berlin, B., Maffi, L., Merrifield, W. R., \& Cook, R. S. (2011). The World Color Survey. Chicago: The university of Chicago Press.

Kay, P., \& Kempton, W. (1984). What is the Sapir-Whorf hypothesis. American Anthropologist, 86, 65-79.

Kay, P., \& Maffi, L. (1999). Color Appearance and the Emergence and Evolution of Basic Color Lexicons. American Anthropologist, 101(4), 743-760.

Kay, P., \& McDaniel, C. K. (1978). The linguistic significance of the meanings of basic color terms. Language, 54(3), 610-646.

Kay, P., \& Regier, T. (2003). Resolving the question of color naming universals. Proceedings of the National Academy of Sciences, 100(15), 9085-9089.

Kay, P., \& Regier, T. (2006). Language, thought and color: recent developments. Trends in Cognitive Sciences, 10(2), 51-54. doi:S1364-6613(05)00353-0 [pii] 10.1016/j.tics.2005.12.007 Kelber, A., \& Osorio, D. (2010). From spectral information to animal colour vision: experiments and concepts. Proceedings of the Royal Society B: Biological Sciences, 277(1688), 1617-1625. doi:rspb.2009.2118 [pii] 10.1098/rspb.2009.2118

Kikutani, M., Roberson, D., \& Hanley, J. R. (2008). What's in the name? Categorical perception for unfamiliar faces can occur through labeling. 15(4), 787-794.

Kim, A. I. (1985). Korean Color Terms: an Aspect of semantic Fields and Related Phenomena. Anthropological Linguistics, 27(4), 425-436

Kim, Y. S., Pak, H., \& Lee, Y. H. (2001). A study on Munsell color space for Korean color names. Journal of Korean Society of Color Studies, 15, 29-36.

Kirby, S., Dowman, M., \& Griffiths, T. L. (2007). Innateness and culture in the evolution of language. Proceedings of the National Academy of Sciences, 104(12), 5241-5245.

doi:0608222104 [pii], 10.1073/pnas.0608222104

Kohonen, O., Parkkinen, J., \& Jaaskelainen, T. (2006). Databases for spectral color science. Color Research and Application, 31(5), 381-390. doi:Doi 10.1002/Col.20244

Krauskopf, J., \& Gegenfurtner, K. R. (1992). Color discrimination and adaptation. Vision Research, 32(11), 2165-2175.

Krauskopf, J., Williams, D. R., \& Heeley, D. W. (1982). Cardinal directions of color space. Vision Research, 22(9), 1123-1131. 


\section{Misconceptions about colour categories}

Kremers, J., Baraas, R. C., \& Marshall, N. J. (Eds.). (2016). Human Color Vision (1 ed.). Cham, Switzerland: Springer International Publishing.

Kuehni, R. G. (2001). Focal colors and unique hues. Color Research \& Application, 26(2), 171 172.

Kuehni, R. G. (2007). Nature and Culture: An Analysis of Individual Focal Color Choices in World Color Survey Languages. Journal of Cognition and Culture, 7(3-4), 151-172. doi:Text Kuehni, R. G. (2014). Unique hues and their stimuli-state of the art. Color Research \& Application, 39(3), 279-287. doi:10.1002/col.21793

Kuriki, I., Lange, R., Muto, Y., Brown, A. M., Fukuda, K., Tokunaga, R., . . Shioiri, S. (2017). The modern Japanese color lexicon. Journal of Vision, 17(3), 1-1. doi:10.1167/17.3.1

Lee, R. L. (1991). What are "all the colors of the rainbow"? Applied Optics, 30(24), 3401-3407. doi:10.1364/AO.30.003401

Lenneberg, E. H. (1953). Cognition in Ethnolonguistics. Language, 29(4), 463-471.

Levinson, S. C. (2000). Yélî Dnye and the theory of basic color terms. Journal of Linguistic Anthropology, 10, 3-55.

Liberman, A. M., Harris, K. S., Hoffman, H. S., \& Griffith, B. C. (1957). The discrimination of speech sounds within and across phoneme boundaries. Journal of Experimental Psychology, 54(5), 358-368.

Lin, H., Luo, M. R., MacDonald, L. W., \& Tarrant, A. W. S. (2001). A cross-cultural colournaming study. Part I: Using an unconstrained method. Color Research \& Application, 26(1), 4060.

Lindsey, D. T., \& Brown, A. M. (2002). Color Naming and the Phototoxic Effects of Sunlight on the Eye. Psychological Science, 13, 506-512.

Lindsey, D. T., \& Brown, A. M. (2006). Universality of color names. Proceedings of the National Academy of Sciences, 103(44), 16608-16613.

Lindsey, D. T., \& Brown, A. M. (2009). World Color Survey color naming reveals universal motifs and their within-language diversity. Proceedings of the National Academy of Sciences USA. doi:0910981106 [pii] 10.1073/pnas.0910981106

Lindsey, D. T., \& Brown, A. M. (2014). The color lexicon of American English. Journal of Vision, 14(2). doi:10.1167/14.2.17

Lindsey, D. T., Brown, A. M., Brainard, D. H., \& Apicella, C. L. (2015). Hunter-Gatherer Color Naming Provides New Insight into the Evolution of Color Terms. Current Biology, 25(18), 24412446. doi:10.1016/j.cub.2015.08.006

Lindsey, D. T., Brown, A. M., Brainard, D. H., \& Apicella, C. L. (2016). Hadza Color Terms Are Sparse, Diverse, and Distributed, and Presage the Universal Color Categories Found in Other World Languages. i-Perception, 7(6), 2041669516681807. doi:10.1177/2041669516681807 Lindsey, D. T., Brown, A. M., Reijnen, E., Rich, A. N., Kuzmova, Y. I., \& Wolfe, J. M. (2010). Color channels, not color appearance or color categories, guide visual search for desaturated color targets. Psychological Science, 21(9), 1208-1214. doi:0956797610379861 [pii]

$10.1177 / 0956797610379861$ 


\section{Misconceptions about colour categories}

Linhares, J. M., Pinto, P. D., \& Nascimento, S. M. (2008). The number of discernible colors in natural scenes. Journal of the Optical Society of America A, 25(12), 2918-2924. doi:173260 [pii] Livingston, K. R., Andrews, J. K., \& Harnad, S. (1998). Categorical perception effects induced by category learning. Journal of Experimental Psychology: Learning, Memory, \& Cognition, 24(3), $732-753$.

Loreto, V., Mukherjee, A., \& Tria, F. (2012). On the origin of the hierarchy of color names. Proceedings of the National Academy of Sciences, 109(18), 6819-6824. doi:10.1073/pnas.1113347109

Lu, A., Hodges, B. H., Zhang, J., \& Wang, X. (2012). A Whorfian speed bump? Effects of Chinese color names on recognition across hemispheres. Language Sciences, 34(5), 591-603. doi:http://dx.doi.org/10.1016/j.langsci.2012.03.014

Lucy, J. A. (1992). Language diversity and thought. Cambridge: Cambridge University Press. Lucy, J. A. (1997a). Linguistic Relativity. Annual review of Anthropology, 26, 291-312. Lucy, J. A. (1997b). The linguistics of color. In C. L. Hardin \& L. Maffi (Eds.), Color categories in thought and language (pp. 320-346). Cambridge: Cambridge University Press.

Lucy, J. A., \& Shweder, R. A. (1979). Whorf and his critics: Linguistic and nonlinguistic influences on color memory. American Anthropologist, 81(3), 581-615.

Lucy, J. A., \& Shweder, R. A. (1988). The Effect of Incidental Conversation on Memory for Focal Colors. American Anthropologist, 90(4), 923-931.

MacAdam, D. L. (1942). Visual Sensitivities to Color Differences in Daylight. Journal of the Optical Society of America, 32(5), 247-274.

MacLaury, R. E. (1997). Ethnographic evidence of unique hues and elemental colors. Behavioral and Brain Sciences, 20(2), 202-203.

MacLaury, R. E., Paramei, G. V., \& Dedrick, D. (2007). Anthropology of color : interdisciplinary multilevel modeling. Amsterdam; Philadelphia: J. Benjamins Pub. Co.

Majid, A., \& Burenhult, N. (2014). Odors are expressible in language, as long as you speak the right language. Cognition, 130(2), 266-270. doi:10.1016/j.cognition.2013.11.004

Malkoc, G., Kay, P., \& Webster, M. A. (2005). Variations in normal color vision. IV. Binary hues and hue scaling. Journal of the Optical Society of America A, 22(10), 2154-2168.

Masaoka, K., Berns, R. S., Fairchild, M. D., \& Moghareh Abed, F. (2013). Number of discernible object colors is a conundrum. Journal of the Optical Society of America A, 30(2), 264-277. doi:10.1364/JOSAA.30.000264

McManus, I. C. (1997). Note: Half-a-Million Basic Colour Words: Berlin and Kay and the Usage of Colour Words in Literature and Science. Perception, 26(3), 367-370. doi:doi:10.1068/p260367 McNeill, N. B. (1972). Color and color terminology. Journal of Linguistics, 8(3), 21-33.

Merrifield, W. R. (1971). Review of: Basic color terms: Their universality and evolution, by Brent Berlin and Paul Kay. Journal of Linguistics, 7, 259-268.

Mills, C. (1984). English color terms: Language, culture, and psychology. Semiotica, 52(1-2), 95110. 


\section{Misconceptions about colour categories}

Miyahara, E. (2003). Focal colors and unique hues. Perceptual and Motor Skills, 97(3 Pt 2), 10381042.

Moore, C., \& Durham, P. J. (Eds.). (1995). Joint attention. Hillsdale, NJ: Lawrence Erlbaum Associates.

Moss, A. E. (1989). Basic Color Terms: Problems and Hypotheses. Lingua, 78, 313-320.

Moss, A. E., Davies, I. R. L., Corbett, G. G., \& Laws, G. (1990). Mapping Russian Basic Color

Terms Using Behavioural Measures. Lingua, 82, 313-332.

Mullen, K. T., \& Kulikowski, J. J. (1990). Wavelength discrimination at detection threshold.

Journal of the Optical Society of America. A, Optics and image science, 7(4), 733-742.

Mylonas, D., \& MacDonald, L. (2016). Augmenting basic colour terms in english. Color Research \& Application, 41(1), 32-42. doi:10.1002/col.21944

Nash, D. (2017). Loss of color terms not demonstrated. Proceedings of the National Academy of Sciences, 114(39), E8131-E8131. doi:10.1073/pnas.1714007114

Newhall, S. M., Nickerson, D., \& Judd, D. B. (1943). Final Report of the O.S.A. Subcommittee on the Spacing of the Munsell Colors. Journal of the Optical Society of America, 33(7), 385-418. Ocelák, R. (2016). “Categorical Perception” and Linguistic Categorization of Color. Review of Philosophy and Psychology, 7(1), 55-70. doi: 10.1007/s13164-015-0237-4

Ogden, C. K., \& Richards, I. A. (1923). The Meaning of Meaning. New York: Harcourt, Brace \& World, Inc.

Olkkonen, M., \& Allred, S. R. (2014). Short-term memory affects color perception in context. PLoS One, 9(1), e86488. doi:10.1371/journal.pone.0086488

Olkkonen, M., McCarthy, P. F., \& Allred, S. R. (2014). The central tendency bias in color perception: effects of internal and external noise. Journal of Vision, 14(11). doi:10.1167/14.11.5

Olkkonen, M., Witzel, C., Hansen, T., \& Gegenfurtner, K. R. (2009). Categorical color constancy for rendered and real surfaces. Journal of Vision(9(8)), 331, 331a.

Olkkonen, M., Witzel, C., Hansen, T., \& Gegenfurtner, K. R. (2010). Categorical color constancy for real surfaces. Journal of Vision, 10(9), 1-22. doi:10.9.16 [pii] 10.1167/10.9.16

Özgen, E. (2004). Language, Learning, and Color Perception. Current Directions in Psychological Science, 13(3), 95-98.

Özgen, E., \& Davies, I. R. L. (1998). Turkish color terms: tests of Berlin and Kay's theory of color universals and linguistic relativity. Linguistics, 36(5), 919-956.

Özgen, E., \& Davies, I. R. L. (2002). Acquisition of Categorical Color Perception: A Perceptual Learning Approach to the Linguistic Relativity Hypothesis. Journal of Experimental Psychology: General, 131(4), 477-493.

Ozturk, O., Shayan, S., Liszkowski, U., \& Majid, A. (2013). Language is not necessary for color categories. Developmental Science, 16(1), 111-115. doi:10.1111/desc.12008

Paggetti, G., Menegaz, G., \& Paramei, G. V. (2016). Color naming in Italian language. Color Research \& Application, 41(4), 402-415. doi:10.1002/col.21953

Pak, H. S., Kim, I. J., Kim, Y. S., \& Lee, M. Y. (2004). An exploratory study on the expressions with Korean color-names and modifiers. Journal of Korean Society of Color Studies, 18, 11-21. 


\section{Misconceptions about colour categories}

Paramei, G. V. (2005). Singing the Russian Blues: An Argument for Culturally Basic Color Terms. Cross-Cultural Research, 39(1), 10-37.

Paramei, G. V., Griber, Y. A., \& Mylonas, D. (2018). An online color naming experiment in Russian using Munsell color samples. Color Research \& Application, 43(3), 358-374.

doi:doi:10.1002/col.22190

Paulsen, G., Uusküla, M., \& Brindle, J. (2016). Color Language and Color Categorization. Newcastle upon Tyne, UK: Cambridge Scholars Publishing.

Philipona, D. L., \& O'Regan, J. K. (2006). Color naming, unique hues, and hue cancellation predicted from singularities in reflection properties. Visual Neuroscience, 23(3-4), 331-339. doi:S0952523806233182 [pii] 10.1017/S0952523806233182

Pilling, M., Wiggett, A., Özgen, E., \& Davies, I. R. L. (2003). Is color "categorical perception" really perceptual? Memory \& Cognition, 31(4), 538-551.

Pitchford, N. J., \& Mullen, K. T. (2002). Is the acquisition of basic-colour terms in young children constrained? Perception, 31(11), 1349-1370.

Pylyshyn, Z. (1999). Is vision continuous with cognition? The case for cognitive impenetrability of visual perception. Behavioural and Brain Sciences, 22(3), 341-365; discussion 366-423.

Regier, T., \& Kay, P. (2009). Language, thought, and color: Whorf was half right. Trends in Cognitive Sciences, 13(10), 439-446 doi:S1364-6613(09)00145-4 [pii]

10.1016/j.tics.2009.07.001

Regier, T., Kay, P., \& Cook, R. S. (2005). Focal colors are universal after all. Proceedings of the National Academy of Sciences, 102(23), 8386-8391. doi:0503281102 [pii]

10.1073/pnas.0503281102

Regier, T., Kay, P., \& Khetarpal, N. (2007). Color naming reflects optimal partitions of color space. Proceedings of the National Academy of Sciences USA, 104(4), 1436-1441. doi:0610341104 [pii] 10.1073/pnas.0610341104

Regier, T., Kemp, C., \& Kay, P. (2015). Word meanings across languages support efficient communication. In B. MacWhinney \& W. O'Grady (Eds.), The handbook of language emergence (pp. 237-263). Hoboken, NJ: Wiley-Blackwell.

Roberson, D. (2005). Color categories are culturally diverse in cognition as well as in language. Cross-Cultural Research, 39(1), 56-71.

Roberson, D., \& Davidoff, J. (2000). The categorical perception of colors and facial expressions: the effect of verbal interference. Memory \& Cognition, 28(6), 977-986.

Roberson, D., Davidoff, J., Davies, I. R. L., \& Shapiro, L. R. (2005). Color categories: evidence for the cultural relativity hypothesis. Cognitive Psychology, 50(4), 378-411. doi:S00100285(04)00076-3 [pii] 10.1016/j.cogpsych.2004.10.001

Roberson, D., Davies, I. R. L., \& Davidoff, J. (2000). Color categories are not universal: replications and new evidence from a stone-age culture. Journal of Experimental Psychology: General, 129(3), 369-398. 


\section{Misconceptions about colour categories}

Roberson, D., \& Hanley, J. R. (2009). Only half right: comment on Regier and Kay. Trends in Cognitive Sciences, 13(12), 500-501; author reply 501. doi:S1364-6613(09)00229-0 [pii] 10.1016/j.tics.2009.10.004

Roberson, D., Hanley, J. R., \& Pak, H. (2009). Thresholds for color discrimination in English and Korean speakers. Cognition, 112(3), 482-487. doi:S0010-0277(09)00139-5 [pii] 10.1016/j.cognition.2009.06.008

Roberson, D., Pak, H., \& Hanley, J. R. (2008). Categorical perception of colour in the left and right visual field is verbally mediated: Evidence from Korean. Cognition, 107(2), 752-762. doi:S0010-0277(07)00235-1 [pii] 10.1016/j.cognition.2007.09.001

Robinson, S. (Writer). (2011). Do You See What I See? In S. Robinson (Producer), Horizon. London: BBC Two.

Rosch Heider, E. (1971). 'Focal' color areas and the development of names. Developmental Psychology, 4, 447-455.

Rosch Heider, E. (1972a). Probabilities, Sampling, and Ethnographic Method. Man, 7(3), 448-466.

Rosch Heider, E. (1972b). Universals in color naming and memory. Journal of Experimental Psychology, 93(1), 10-20.

Sandford, J. L. (2012). Blu, azzurro, celeste- What color is blue for Italian speakers compared to English speakers? In M. Rossi (Ed.), Colour and Colorimetry. Multidisciplinary Contributions. Vol. VIII B (pp. 281-288).

Sapir, E. (1912). Language and Environment. American Anthropologist, 14(2), 226-242.

Saunders, B. A. C. (1995). Disinterring Basic Color Terms: a study in the mystique of cognitivism. History of the Human Sciences, 8(4), 19-38.

Saunders, B. A. C. (2000). Revisiting Basic Color Terms. Journal of the Royal Anthropological Institute, 6(1), 81-99.

Saunders, B. A. C. (2007). The Debate about Colour Naming in 19th Century German Philology. Leuven, Belgium.

Saunders, B. A. C., \& van Brakel, J. (1997). Are there non-trivial constraints on colour categorization? Behavioral and Brain Sciences, 20(2), 167-228.

Schneider, R. U. (2002, 10/02). Das Experiment -- Die Eskimos und der Schnee. Neue Züricher Zeitung.

Schouten, B., Gerrits, E., \& van Hessen, A. (2003). The end of categorical perception as we know it. Speech Communication, 41, 71-80.

Self, E. M. (2014). Unique Hues. In R. Luo (Ed.), Encyclopedia of Color Science and Technology (pp. 1-4). New York, NY: Springer New York.

Shevell, S. K. (2003). The science of color (2 ed.). Oxford, UK: Elsevier Science.

Shevell, S. K., \& Martin, P. R. (2017). Color opponency: tutorial. Journal of the Optical Society of America A, 34(7), 1099-1108. doi:10.1364/JOSAA.34.001099

Shinoda, H., Uchikawa, K., \& Ikeda, M. (1993). Categorized color space on CRT in the aperture and the surface color mode. Color Research \& Application, 18(5), 326-333. 


\section{Misconceptions about colour categories}

Skelton, A. E., Catchpole, G., Abbott, J. T., Bosten, J. M., \& Franklin, A. (2017). Biological origins of color categorization. Proceedings of the National Academy of Sciences, 114(21), 55455550. doi:10.1073/pnas.1612881114

Smithson, H. E., Anderson, P. S., Dinkova-Bruun, G., Fosbury, R. A., Gasper, G. E., Laven, P., . . . Tanner, B. K. (2014). Color-coordinate system from a 13th-century account of rainbows. J Opt Soc Am A Opt Image Sci Vis, 31(4), A341-349. doi:10.1364/JOSAA.31.00A341

Snow, D. L. (1971). Samoan Color Terminology a Note on the Universality and Evolutionary Ordering of Color Terms. Anthropological Linguistics, 13(8), 385-390

Steels, L., \& Belpaeme, T. (2005). Coordinating perceptually grounded categories through language: A case study for colour. Behavioral and Brain Sciences, 28, 469-529.

Stockman, A., \& Sharpe, L. T. (2000). The spectral sensitivities of the middle- and longwavelength-sensitive cones derived from measurements in observers of known genotype. Vision Research, 40(13), 1711-1737. doi:S0042-6989(00)00021-3 [pii]

Sturges, J., \& Whitfield, T. W. A. (1997). Salient features of Munsell colour space as a function of monolexemic naming and response latencies. Vision Research, 37(3), 307-313. doi:S00426989(96)00170-8 [pii]

Suegami, T., Aminihajibashi, S., \& Laeng, B. (2014). Another look at category effects on colour perception and their left hemispheric lateralisation: no evidence from a colour identification task. Cognitive processing, 15(2), 217-226. doi:10.1007/s10339-013-0595-8

Taft, C., \& Sivik, L. (1997). Salient Color Terms in Four Languages. Scandinavian Journal of Psychology, 38(1), 29-34.

Thierry, G., Athanasopoulos, P., Wiggett, A., Dering, B., \& Kuipers, J.-R. (2009). Unconscious effects of language-specific terminology on preattentive color perception. Proceedings of the National Academy of Sciences USA, 106(11), 4567-4570. doi:0811155106 [pii] 10.1073/pnas.0811155106

Tyson, R. E. (1998). Color Naming and Color Categorization in Korean. Japanese/Korean Linguistics, 7, 177-196.

Uchikawa, H., Uchikawa, K., \& Boynton, R. M. (1989). Influence of achromatic surrounds on categorical perception of surface colors. Vision Research, 29(7), 881-890.

Uchikawa, K. (2014). Categorical color perception of color normal and deficient observers. Optical Review, 21(6), 911-918. doi:10.1007/s10043-014-0144-4

Uchikawa, K., \& Boynton, R. M. (1987). Categorical color perception of Japanese observers: comparison with that of Americans. Vision Research, 27(10), 1825-1833.

Valberg, A. (2001). Unique hues: an old problem for a new generation. Vision Research, 41(13), 1645-1657. doi:S0042-6989(01)00041-4 [pii]

Vazquez-Corral, J., O'Regan, J. K., Vanrell, M., \& Finlayson, G. D. (2012). A new spectrally sharpened sensor basis to predict color naming, unique hues, and hue cancellation. Journal of Vision, 12(6), 7. doi:10.1167/12.6.7

Vox. (2016). The surprising pattern behind color names around the world. Retrieved from https://www.youtube.com/watch?v=gMqZR3pqMjg 


\section{Misconceptions about colour categories}

Webster, M. A., \& Kay, P. (2007). Individual and Population Differences in Focal Colors. In R. E. MacLaury, G. V. Paramei, \& D. Dedrick (Eds.), Anthropology of Color. Amsterdam: John Benjamins.

Webster, M. A., \& Kay, P. (2012). Color categories and color appearance. Cognition, 122(3), 375392. doi:S0010-0277(11)00279-4 [pii]; 10.1016/j.cognition.2011.11.008

Webster, M. A., Miyahara, E., Malkoc, G., \& Raker, V. E. (2000). Variations in normal color vision. II. Unique hues. Journal of the Optical Society of America A, 17(9), 1545-1555.

Weiss, D., Witzel, C., \& Gegenfurtner, K. (2017). Determinants of Colour Constancy and the Blue Bias. i-Perception, 8(6), 2041669517739635. doi:10.1177/2041669517739635

Welsch, N., \& Liebmann, C. C. (2004). Farben. Natur - Technik - Kunst. Heidelberg: Spektrum Akademischer Verlag.

Wescott, R. W. (1970). Bini Color Terms. Anthropological Linguistics, 12(9), 349-360.

Whorf, B. L. (1956). Language, Thought and Reality: Selected Writings of Benjamin Lee Whorf. Cambridge, MA: MIT Press.

Winawer, J., \& Witthoft, N. (2014). Effect of Color Terms on Color Perception. In R. Luo (Ed.), Encyclopedia of Color Science and Technology (pp. 1-9). Berlin, Heidelberg: Springer Berlin Heidelberg.

Winawer, J., Witthoft, N., Frank, M. C., Wu, L., Wade, A. R., \& Boroditsky, L. (2007). Russian blues reveal effects of language on color discrimination. Proceedings of the National Academy of Sciences USA, 104(19), 7780-7785. doi:0701644104 [pii] 10.1073/pnas.0701644104

Witzel, C. (2012). Colours' appearance in the light of language and experience. (Dr. rer. nat. Dissertation), Justus-Liebig-Universität, Gießen.

Witzel, C. (2016). New Insights Into the Evolution of Color Terms or an Effect of Saturation? iPerception, 7(5), 1-4. doi:10.1177/2041669516662040

Witzel, C. (in press). The role of saturation in colour naming and colour appearance. In G.

Paramei, C. P. Biggam, \& L. MacDonald (Eds.), Colour Studies: A broad spectrum. Amsterdam: John Benjamin Publishing Company.

Witzel, C., Cinotti, F., \& O'Regan, J. K. (2015). What determines the relationship between color naming, unique hues, and sensory singularities: Illuminations, surfaces, or photoreceptors? Journal of Vision, 15(8), 19. doi:10.1167/15.8.19

Witzel, C., Flack, Z., \& Franklin, A. (2013, 8-12. July 2013). Categorical colour constancy during colour term acquisition. Paper presented at the AIC2013 - 12th international AIC congress, Newcastle upon Tyne, UK.

Witzel, C., \& Franklin, A. (2014). Do focal colors look particularly "colorful"? Journal of the Optical Society of America. A, Optics, image science, and vision, 31(4), A365-374.

doi:10.1364/JOSAA.31.00A365

Witzel, C., \& Gegenfurtner, K. R. (2011). Is there a lateralized category effect for color? Journal of Vision, 11(12), 16. doi:10.1167/11.12.16

Witzel, C., \& Gegenfurtner, K. R. (2013). Categorical sensitivity to color differences. Journal of Vision, 13(7). doi:10.1167/13.7.1 


\section{Misconceptions about colour categories}

Witzel, C., \& Gegenfurtner, K. R. (2014). Category effects on colour discrimination. In W. Anderson, C. P. Biggam, C. A. Hough, \& C. J. Kay (Eds.), Colour Studies: A broad spectrum (pp. 200-211). Amsterdam: John Benjamin Publishing Company.

Witzel, C., \& Gegenfurtner, K. R. (2015). Categorical facilitation with equally discriminable colors. Journal of Vision, 15(8), 22. doi:10.1167/15.8.22

Witzel, C., \& Gegenfurtner, K. R. (2016). Categorical perception for red and brown. Journal of Experimental Psychology: Human Perception \& Performance, 42(4), 540-570.

doi:10.1037/xhp0000154

Witzel, C., \& Gegenfurtner, K. R. (2018, in press). Are red, yellow, green, and blue perceptual categories? Vision Research. doi:https://doi.org/10.1016/j.visres.2018.04.002

Witzel, C., \& Gegenfurtner, K. R. (in press). Color Perception: Objects, constancy and categories. Annual Review of Vision Science, 4.

Witzel, C., \& Hammermeister, J. (2017). Typical and unique hues depend on saturation. Paper presented at the ICVS2017, Erlangen, Germany.

Witzel, C., Hansen, T., \& Gegenfurtner, K. R. (2008). Wie sich Farben mit den Betrachtern und mit den Zeiten ändern. Paper presented at the Tagung experimentell arbeitender Psychologen (TeaP), Marburg.

Witzel, C., Maule, J., \& Franklin, A. (2013). Focal colors as perceptual anchors of color categories. Journal of Vision, 13(9 (VSS abstracts)), 1164-1164. doi:10.1167/13.9.1164 Witzel, C., Maule, J., \& Franklin, A. (under revision). Are red, yellow, green and blue particularly "colorful"?

Witzel, C., Olkkonen, M., \& Gegenfurtner, K. R. (2016). Memory colours affect colour appearance. Behavioral and Brain Sciences, 39, 51-52. doi:10.1017/S0140525X15002587 Witzel, C., Olkkonen, M., \& Gegenfurtner, K. R. (2018). A Bayesian Model of the Memory Colour Effect. Iperception, 9 (2), 1-16. doi:10.1177/2041669518771715

Witzel, C., van Alphen, C., Godau, C., \& O'Regan, J. K. (2016). Uncertainty of sensory signal explains variation of color constancy. Journal of Vision, 16(15), 8. doi:10.1167/16.15.8

Wolff, P., \& Holmes, K. J. (2011). Linguistic relativity. Wiley Interdisciplinary Reviews: Cognitive Science, 2(3), 253-265. doi:10.1002/wcs.104

Wright, W. D., \& Pitt, F. H. G. (1934). Hue Discrimination in normal colour-vision. Proceedings of the Physical Society of London, 46, 459-473.

Wuerger, S. M., Maloney, L. T., \& Krauskopf, J. (1995). Proximity judgments in color space: tests of a Euclidean color geometry. Vision Research, 35(6), 827-835. doi:0042-6989(94)00170-Q [pii] Wuerger, S., \& Xiao, K. (2014). Color Vision, Opponent Theory. In R. Luo (Ed.), Encyclopedia of Color Science and Technology (pp. 1-6). Berlin, Heidelberg: Springer Berlin Heidelberg.

Yang, J., Kanazawa, S., \& Yamaguchi, M. K. (2013). Can Infants Tell the Difference between Gold and Yellow? PLoS One, 8(6), e67535. doi:10.1371/journal.pone.0067535

Yendrikhovskij, S. N. (2001). A computational model of colour categorization. Color Research \& Application, 26(S1), S235-S238. 


\section{Misconceptions about colour categories}

Young, T. (1802). II. The Bakerian Lecture. On the theory of light and colours. Philosophical Transactions of the Royal Society of London, 92, 12-48. doi:10.1098/rstl.1802.0004

Zhou, K., Mo, L., Kay, P., Kwok, V. P. Y., Ip, T. N. M., \& Tan, L. H. (2010). Newly trained lexical categories produce lateralized categorical perception of color. Proceedings of the National Academy of Sciences USA, 107(22), 9974-9978. doi:1005669107 [pii] 10.1073/pnas.1005669107 Zimmer, A. C. (1982). What Really is Turquoise? A Note on the Evolution of Color Terms. Psychological Research, 44, 213-230.

Zollinger, H. (1988). Categorical color perception: influence of cultural factors on the differentiation of primary and derived basic color terms in color naming by Japanese children. Vision Research, 28(12), 1379-1382.

Zollinger, H. (1984). Why just turquoise? Remarks on the evolution of color terms. Psychological Research, 46(4), 403-409. 